\title{
The effect of a low-viscosity near-wall film on bypass transition in boundary layers
}

\author{
SEO YOON JUNG AND TAMER A. ZAKI $\dagger$ \\ Department of Mechanical Engineering, Imperial College, London SW7 2AZ, UK \\ (Received ?; revised ?; accepted ?. - To be entered by editorial office)
}

Bypass transition in a two-fluid boundary layer is examined using direct numerical simulations. A less viscous wall film is considered and the impact on transition location is evaluated at two different viscosity ratios and free-stream turbulence intensities. The less viscous wall film absorbs the mean shear from the outer stream, weakens the lift-up mechanism, and alters the disturbance field inside the boundary layer. These effects all favour a delay in the onset of bypass transition. However, the viscosity and mean-shear discontinuities across the two-fluid interface introduce a new mechanism for the generation of wall-normal vorticity in the boundary layer, and can therefore promote transition to turbulence. Conditionally-averaged statistics and streak tracking techniques are adopted in order to examine the impact of the wall film on the bypass transition process. It is shown that the weaker amplification of the streaks in the outer fluid can delay breakdown to turbulence, despite the additional disturbance generation at the two-fluid interface. The efficacy of the wall film in delaying transition is demonstrated at moderate level of free-stream turbulence intensity, but is reduced as the turbulence intensity is increased.

Key words: bypass transition; two-fluid boundary layer; direct numerical simulation

\section{Introduction}

The paths to boundary layer turbulence are diverse and have inspired a great deal of research. Even in canonical flow configurations such as a zero-pressure-gradient boundary layer, breakdown to turbulence can follow various routes: When the background perturbation levels are inappreciable, transition is governed by the evolution of discrete instability waves (Kleiser \& Zang 1991). However, with a slight increase in the level of environmental disturbances, a shorter path termed 'bypass transition' leads to boundary layer turbulence at lower Reynolds numbers (for a recent review, see Zaki 2013). The current work considers the influence of introducing a wall film of different viscosity on bypass transition. The addition of a different fluid can introduce new instability mechanisms, and alter the bypass transition process. However, appropriate choice of the film properties can have a favourable effect in terms of overall flow stability and transition delay. The current state of our understanding of bypass transition beneath free-stream turbulence is summarized and, where possible, the discussion addresses the influence of introducing a wall film of different viscosity.

$\dagger$ Present address: Department of Mechanical Engineering, Johns Hopkins University, Baltimore, MD 21218, USA. Email address for correspondence: t.zaki@jhu.edu 


\subsection{Free-stream perturbations}

The initial stage of bypass transition concerns the interaction of free-stream vortical disturbances with the boundary layer. In the inviscid limit, free-stream disturbances decay exponentially at the boundary-layer edge due to the influence of the mean shear, and this phenomenon is now known as shear sheltering (Hunt \& Durbin 1999). At finite Reynolds number, however, the sheltering mechanism is less effective, and low-frequency disturbances from the free stream can cause a finite distortion within the boundary layer (Jacobs \& Durbin 1998). Following a similar approach to Charru \& Hinch (2000), Zaki \& Saha (2009) provided a physical interpretation. They compared the diffusive and convective timescales of vortical waves at the edge of the boundary layer. Three regimes were identified based on the ratio of these two timescales, $k_{x} U^{\prime} \delta / \nu k_{y}^{2}$, where $k_{x}$ and $k_{y}$ are the streamwise and wall-normal disturbance wave numbers, $U^{\prime}$ is the mean shear, $\delta$ is the boundary layer $99 \%$ thickness and $\nu$ is the fluid viscosity. For high values of this ratio, the inviscid shear-sheltering behaviour is recovered. For small values, lowfrequency vortical disturbances can penetrate the boundary layer. In the intermediate regime, where this parameter is on the order of unity, the influence of the vortical waves decays with distance into the shear.

The same problem was also examined in the context of two-fluid boundary layers (Charru \& Hinch 2000; Zaki \& Saha 2009). Since the filtering of disturbances by the shear is an inviscid phenomenon, it is intuitive to expect that a lower-viscosity wall film would enhance stability. However, the analysis showed that a lower-viscosity film initially enhances the signature of outer vortical disturbances inside the near-wall film (Zaki \& Saha 2009). Only after the ratio of the film to the free-stream viscosities is reduced beyond a critical value does shear sheltering become effective. In light of this result, and the additional risk of introducing new two-fluid interfacial instabilities (Yih 1967; Hooper \& Boyd 1987; Ó Náraigh et al. 2011a,b), it should be cautioned that naively inserting a lower-viscosity wall film does not guarantee enhanced stability of the boundary layer.

\subsection{The primary boundary-layer disturbance}

Forcing due to low-frequency perturbations generates an energetic response within the boundary layer in the form of streamwise-elongated streaks, or Klebanoff distortions. The amplification of streaks is due to an inviscid lift-up mechanism whereby quasi-streamwise vortices lift low-velocity fluid away from the wall and sweep high-velocity fluid towards the wall. The energy of the streaks is proportional to the distance from the leading edge (Westin et al. 1994). In the absence of high-frequency background disturbances, the streaks do not break down to turbulence (Zaki \& Durbin 2005, 2006). Instead they decay due to viscosity. The transient growth of boundary-layer streaks is now well characterized in the context of single-fluid flows (e.g. Butler \& Farrell 1992; Luchini 2000). In comparison, the literature on the amplification of streaks in two-fluid boundary layers is scarce. Furthermore, the presence of the interface in a two-fluid boundary layer can introduce a different primary instability. Yih (1967) showed the existence of a long-wavelength interfacial instability due to the viscosity mismatch at the interface. A short-wavelength instability emerges at high Reynolds number when the viscosity of the lower fluid is less than that of the upper fluid (Hooper \& Boyd 1987). The present study exploits the thin layer effect which stabilizes the interfacial mode when the film viscosity is lower than the outer stream (Renardy 1987; Charru \& Hinch 2000). The stabilization of the interfacial mode, however, addresses the long-time asymptotic behavior of the system. In the transient response of the flow, even a stable interfacial mode can contribute to short-time energy amplification. Examples which demonstrate this phenomenon include the work by Yecko \& Zaleski (2005) in two-fluid mixing layers, and by Malik \& Hooper (2007) 
in two-layer Poiseuille flow. These studies support the view that transient disturbance growth can play an important role in two-fluid shear flows, including boundary layers.

\subsection{Breakdown to turbulence}

Once the primary boundary-layer instability reaches a high amplitude, it becomes vulnerable to secondary instability and breakdown to turbulence. In the context of bypass transition, these are instabilities of the streaky base flow. For example, Matsubara \& Alfredsson (2001) provided a detailed time-series that support this view. Using direct numerical simulation (DNS), Jacobs \& Durbin (2001) showed that breakdown occurs when the lifted low-speed streaks are buffeted by the high-frequency small scales of turbulence in the free stream. Their observations were confirmed experimentally, for example by Hernon, Walsh \& McEligot (2007) and Mandal et al. (2010), and complemented by a number of computational studies (e.g. Schlatter et al. 2008). In addition to the streak breakdown scenario which occurs near the edge of the boundary layer, Nagarajan et al. (2007) identified another mechanism that originates near the wall and is initiated by the amplification of an instability wavepacket.

In order to explain the numerical and experimental observations of breakdown, a number of studies evaluated the secondary instability of the streaky boundary-layer flow. Andersson et al. (2001) performed an inviscid instability analysis of the linearly-optimal streaks, and could only predict the instability near the edge of the boundary layer. Vaughan \& Zaki (2011) applied Floquet theory to examine the secondary instability of unsteady streaks. Their analysis predicted two types of instability modes: an outer mode which resides near the edge of the boundary layer and an inner instability near the wall. These two studies focused on idealized base states where the streaks form a repeated pattern in the span. More recently, Hack \& Zaki (2014) established a connection between linear stability theory and localized breakdown of realistic streaks in bypass transition beneath free-stream turbulence. They performed direct stability analyses of streaky boundary-layer profiles extracted from DNS and demonstrated that linear theory can accurately predict the localized streak instabilities that precede the inception of turbulence spots. Since the two-fluid configuration has not been studied before, it is not possible to anticipate the mode of secondary instability and breakdown to turbulence. However, the earlier work on the single-fluid flow provides a background against which the simulations performed herein can be analyzed.

\subsection{Objectives and outline}

Significant advancement in the study of bypass transition in single-fluid boundary layers has been possible due to recent experimental, numerical and theoretical efforts. In comparison, relatively little is known regarding bypass transition in two-fluid boundary layers or, equivalently, the influence of a wall film on bypass breakdown. In the current study, direct numerical simulations are performed to examine transition in two-fluid boundary layers beneath free-stream turbulence.

A schematic of the simulation setup is shown in figure 1. The simulations are performed for two moderate levels of free-stream turbulence intensity, $T u=2.5 \%$ and $3.5 \%$. This choice is motivated by the literature, where the location of bypass transition is known to become progressively less sensitive to the free-stream condition at higher $T u$. The two fluids are immiscible and, while they share the same density, they have different viscosities with the wall film being less viscous. The film thickness and viscosity are important parameters that influence the stability of the flow. The main considerations in

their selection are to reduce the amplification of streaks and to avoid the generation of new instabilities. The former objective favours a thick low-viscosity film while the latter 


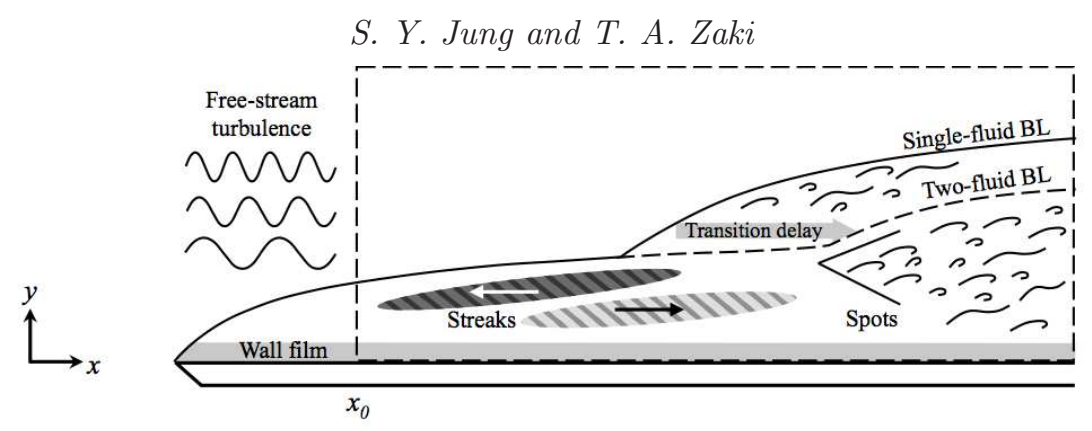

FiguRE 1. Schematic of bypass transition in a two-fluid boundary layer.

requires a thin film with smaller viscosity mismatch. Three viscosity ratios are considered, $\mu_{B T}\left(\equiv \mu_{B} / \mu_{T}\right)=1.0,0.5$ and 0.2 , where subscripts $T$ and $B$ denote the top (outer) and bottom (wall film) fluids, respectively. The height of the wall film at the inlet is $10 \%$ of the reference boundary-layer thickness, $\delta_{0}$, which is the $99 \%$ thickness in the matched viscosity flow. These properties are selected based on precursor linear analyses (Zaki \& Saha 2009; Saha, Jung \& Zaki 2010), and their impact on transition will be discussed in detail.

This paper is organized as follows. A description of the numerical method and the inflow condition is provided in $\S 2$. Reynolds-averaged statistics are provided in $\S 3$, followed by analysis of instantaneous and conditionally-sampled fields in $\S 4$. Finally, a brief discussion of the higher inlet turbulence intensity is presented in $\S 5$.

\section{Numerical method}

\subsection{Governing equations and simulation parameters}

The continuity and Navier-Stokes equations for incompressible flow of two immiscible fluids with matching densities but different viscosities are,

$$
\begin{gathered}
\frac{\partial u_{j}}{\partial x_{j}}=0 \\
\frac{\partial u_{i}}{\partial t}+\frac{\partial u_{i} u_{j}}{\partial x_{j}}=-\frac{\partial p}{\partial x_{i}}+\frac{1}{R e_{\delta_{0}}} \frac{\partial}{\partial x_{j}}\left[\mu\left(\frac{\partial u_{i}}{\partial x_{j}}+\frac{\partial u_{j}}{\partial x_{i}}\right)\right] .
\end{gathered}
$$

Terms in the above equations are non-dimensionalized using the free-stream velocity $U_{\infty}$ and the single-fluid boundary layer thickness at the inlet $\delta_{0}$. The viscosity, $\mu$, is normalized by its value in the top fluid. The ratio of the bottom to the top fluid viscosity is denoted $\mu_{B T}$. The Reynolds number in the equations is defined as $R e_{\delta_{0}}\left(\equiv \rho U_{\infty} \delta_{0} / \mu_{T}\right)=800$. The velocity components in the streamwise $(x)$, wall-normal $(y)$ and spanwise $(z)$ directions are $u, v$ and $w$, respectively, and the pressure is $p$. Hereafter, uppercase symbols refer to mean quantities, and primed symbols, such as $u^{\prime}$, are reserved for fluctuations.

The Navier-Stokes equations were solved on a staggered grid with a local-volumeflux formulation (Rosenfeld, Kwak \& Vinokur 1991). The viscous terms were integrated in time implicitly using Crank-Nicolson method and the convective terms were treated explicitly using Adams-Bashforth scheme. The overall accuracy of the present numerical method is second order in space and time. By applying the fractional step algorithm to the continuity and Navier-Stokes equations, a three-step time-advancement scheme is 


\begin{tabular}{ccccccc}
\hline$T u(\%)$ & $L_{x} \times L_{y} \times L_{z}$ & No. of grid points & $\Delta x^{+}$ & $\Delta y_{\text {min }}^{+}$ & $\Delta z^{+}$ & $\Delta t^{+}$ \\
2.5 & $1200 \times 40 \times 30$ & $4097 \times 193 \times 193$ & 10.48 & 0.36 & 5.59 & 0.016 \\
3.5 & $600 \times 40 \times 30$ & $2049 \times 193 \times 193$ & 10.99 & 0.38 & 5.86 & 0.018
\end{tabular}

TABLE 1. Summary of simulation parameters, and the spatial and temporal resolutions.

obtained,

$$
\begin{gathered}
\frac{\hat{u}_{i}-u_{i}{ }^{n}}{\Delta t}=\frac{1}{2}\left(3 N_{i}{ }^{n}-N_{i}{ }^{n-1}\right)+\frac{1}{2} \frac{1}{R e_{\delta_{0}}} \frac{\partial}{\partial x_{j}}\left[\mu \frac{\partial}{\partial x_{j}}\left(\hat{u}_{i}+u_{i}{ }^{n}\right)\right]-\frac{\partial \Phi^{n}}{\partial x_{i}}, \\
\frac{\partial^{2} \Delta \Phi}{\partial x_{j} \partial x_{j}}=\frac{1}{\Delta t} \frac{\partial \hat{u}_{j}}{\partial x_{j}}, \\
\frac{u_{i}^{n+1}-\hat{u}_{i}}{\Delta t}=-\frac{\partial \Delta \Phi}{\partial x_{i}},
\end{gathered}
$$

where $N$ is the discrete operator for the convective terms, and $\Delta t$ is the computational time step. Here, $\Phi$ and $\Delta \Phi$ are the pseudo-pressure and its time difference $\left(\Phi^{n+1}-\Phi^{n}\right)$, respectively. By adopting the $\Delta \Phi$ form of the discrete Navier-Stokes equations, we enforce the appropriate boundary conditions on the velocity field directly without the need to incorporate the pressure gradient term (Kim \& Moin 1985).

Generally, the solution to the pressure equation is computationally expensive due to the elliptic nature of the problem, and this issue is exacerbated in the case of variable fluid properties (van der Vorst 2003). For example, in the general two-fluid case where the density varies across the interface, we solve the Poisson equation using Bi-Conjugate Gradient Stabilized (BiCGStab) method (van der Vorst 1992) with a three-dimensional multigrid pre-conditioner. The Krylov-based method is advantageous for fast convergence of the solution in the presence of the jump in shear across the two-fluid interface. In the matched density case which we consider here, a significant computational advantage is achieved by applying a Fourier Transform in the span and converting the pressure Poisson equation into a Helmholtz equation.

The convective outflow condition $\partial u / \partial t+c \partial u / \partial x=0$ was applied at the outlet where $c$ is the local bulk velocity. The no-slip condition was imposed at the bottom wall. The top boundary conditions were $\partial u / \partial y=\partial w / \partial y=0$ and $v=-\int \partial u / \partial x d y$. Periodic boundary conditions were applied in the spanwise direction.

Two free-stream turbulence intensities were simulated, $T u=2.5 \%$ and $3.5 \%$. The sizes of the computational domains and the number of grid points are provided in table 1 . The computational domain is a rectangular box with a sufficiently large spanwise extent in order to avoid spurious correlations. For the lower turbulence intensity case, the length of computational domain is longer, $2.6 \times 10^{4}<R e_{x}\left(\equiv \rho U_{\infty} x / \mu_{T}\right)<9.9 \times 10^{5}$, in order to capture the full transition process. The grid is clustered in the wall-normal direction, and is uniformly distributed in the streamwise and spanwise directions. Table 1 also reports the grid resolution and the computational time-step in wall units scaled using the maximum friction velocity, $\max _{x} u_{\tau}(x)$, from the single-fluid computation which provides a conservative estimate. The computational time-step was $0.01 \delta_{0} / U_{\infty}$. Beyond the initial transient, the total averaging time was $2,400 \delta_{0} / U_{\infty}$ time units. Simulations of the twofluid boundary layers are particularly challenging due the computational requirements associated with tracking the interface as discussed below. 


\subsection{Level set method}

The level set method introduced by Osher \& Sethian (1988) has been widely used in simulations of multiphase flows. This method is based on an implicit representation of the interface for which the equation of motion is,

$$
\frac{\partial \phi}{\partial t}+\frac{\partial u_{j} \phi}{\partial x_{j}}=0
$$

where $\phi$ is the level set function. In the conventional level set technique, a signed distance function depicting the interface as $\phi=0$ is used. Advantages of the level set method include automatic handling of topological changes, efficient parallelization as well as simplicity of evaluating geometric quantities. However, one of the main limitations of this method is poor mass conservation. In order to overcome this difficulty, we adopted the conservative level set method proposed by Desjardins, Moureau \& Pitsch (2008). This method ensures accurate and robust interface transport by using a hyperbolic tangent function,

$$
\psi=\frac{1}{2}\left(\tanh \left(\frac{\phi}{2 \epsilon}\right)+1\right),
$$

where $\epsilon \equiv 0.5 \min (\Delta x, \Delta y, \Delta z)$ is a parameter which sets the thickness of the interface. The interface location is now portrayed by the iso-surface $\psi=0.5$. The transport equation for $\psi$ corresponding to the advection of the interface is,

$$
\frac{\partial \psi}{\partial t}+\frac{\partial u_{j} \psi}{\partial x_{j}}=0
$$

Due to the sharp change in $\psi$ across the interface, the above equation accurately approximates the material derivative of fluid properties, such as $D \mu / D t=0$.

In the presence of sharp gradients of velocity and pressure across the interface, it is computationally very challenging to maintain $\psi$ as a non-oscillatory function. Distorted $\psi$ incurs unphysical oscillations. The difficulty is attributed to the nature of the Hamilton-Jacobi-type equation (2.8) (Sethian \& Smereka 2003). In order to maintain the smoothness of $\psi$, we introduced the re-initialization equation,

$$
\frac{\partial \psi}{\partial \tau}+\nabla \cdot(\psi(1-\psi) \mathbf{n})=\nabla \cdot(\epsilon(\nabla \psi \cdot \mathbf{n}) \mathbf{n})
$$

where $\tau$ and $\mathbf{n} \equiv \nabla \psi /|\nabla \psi|$ are the pseudo-time step and the interface normal vector, respectively. The above equation is composed of a compression term on the left-hand side which sharpens the interface, and a diffusion term on the right hand side which maintains numerical stability. Solution of equation (2.9) is repeated until the steady state is reached. Typically, 3 or 4 iterations were required for the convergence of the solution by setting $\Delta \tau=\epsilon$. The physical properties of two-fluids are computed from,

$$
\mu=\mu_{B T}+\left(1-\mu_{B T}\right) \psi .
$$

The third-order total variation diminishing (TVD) Runge-Kutta scheme (Shu \& Osher 1988) was used for time integration of equations (2.8) and (2.9). To accurately resolve the sharp interface profile, a 5th-order upstream central scheme (Nourgaliev \& Theofanous 2007) was employed for the convective term of equation (2.8). The compression and diffusion terms in equation (2.9) were spatially discretized by second-order central differencing.

The computations of equations (2.8) and (2.9) for the entire domain are inefficient owing to the interface movement within a confined region. In order to reduce the computational effort, an adaptive narrow-band approach was exploited (Peng et al. 1999). In 
this method, a narrow band formed by a few grid cells around the interface is introduced using a mask function. The mask function identifies the grid points within the narrow band and then the level-set and re-initialization equations are solved at these points. In addition, a multi-level mesh refinement (MMR) for the level-set function was utilized to improve the spatial resolution of the interface transport. In this approach, the flow field is solved at the coarsest mesh and the level set function is evaluated at the finest grid. The multigrid hierarchy with three levels was selected in the $x$ and $z$ directions. There was no additional multi-level grid refinement in the $y$ direction.

The present level-set algorithm has been validated extensively by comparison to linear and non-linear stability results (see Cheung \& Zaki 2011). In addition, two canonical interface tracking problems were also examined: the transport of Zalesak's disk (Zalesak 1979) and the rise of an air bubble in quiescent water (Gueyffier et al. 1999; Yang \& Stern 2009). In the case of the Zalesak's disk, the size of the computational domain was $\left(L_{x}, L_{y}\right)=(100,100)$ and the number of grid points was $\left(N_{x}, N_{y}\right)=(257,257)$. Solid-body rotation, $u=(\pi / 314)(50-y)$ and $v=(\pi / 314)(x-50)$, was prescribed to the disk for ten revolutions and the error in the interface location was evaluated using the $L_{1}$-norm,

$$
\varepsilon \equiv \frac{1}{L} \int\left|H\left(\psi_{\text {exact }}\right)-H\left(\psi_{\text {computed }}\right)\right| \mathrm{d} x \mathrm{~d} y,
$$

where $L$ is the perimeter of the expected interface and the indicator function $H=\{0,1\}$ distinguishes the inner and outer regions of the disk. After one, two, five and ten full rotations, the errors were $\varepsilon=0.028,0.032,0.042$ and 0.062 , respectively. For the rising bubble, the size of the computational domain was $\left(L_{x}, L_{y}, L_{z}\right)=(12,24,12) R$ where $R$ is the radius of the initially-spherical bubble. The number of grid points in each direction was $\left(N_{x}, N_{y}, N_{z}\right)=(129,257,129)$. The viscosity and density ratios of air to water were prescribed, $\mu_{a} / \mu_{w}=0.085$ and $\rho_{a} / \rho_{w}=0.0011$, and the terminal velocity was chosen as the reference scale. The Reynolds, Froude and Weber numbers were $R e=9.8$, $F r=0.8718$ and $W e=7.6$. The bubble was released at an initial position $(x / R, y / R)=$ $(6.0,1.5)$. As the bubble rose, its deformation, vertical displacement and rising speed agreed with the results by Gueyffier et al. (1999) and Yang \& Stern (2009).

\subsection{Inflow generation}

The base flow at the inlet to the computational domain is a two-fluid boundary layer $\mathbf{U}=\left[U_{j}(y), V_{j}(y), 0\right]$ with density $\rho$ and dynamic viscosities $\mu_{j}$. The subscript $j=\{T, B\}$ denotes the top and bottom fluids, respectively. The base flow is obtained by solving the following equation in boundary layer coordinates (Nelson, Alving \& Joseph 1995),

$$
F \frac{\partial^{2} F}{\partial \zeta^{2}}+\nu_{j} \frac{\partial^{3} F}{\partial \zeta^{3}}-\chi\left(\frac{\partial F}{\partial \zeta} \frac{\partial}{\partial \chi}\left(\frac{\partial F}{\partial \zeta}\right)-\frac{\partial^{2} F}{\partial \zeta^{2}} \frac{\partial F}{\partial \chi}\right)=0
$$

where $\nu_{j}=\mu_{j} / \rho, \chi \equiv \sqrt{U_{\infty} x /\left(2 \nu_{T}\right)}, \zeta \equiv y \sqrt{U_{\infty} /\left(2 \nu_{T} x\right)}$ and $F \equiv \Psi / \sqrt{2 \nu_{T} U_{\infty} x}$. Here, $\Psi$ and $x$ are the streamfunction and the downstream location from the leading edge, respectively. The interface height $\zeta^{*}$ is governed by the kinematic condition:

$$
\frac{\partial \zeta^{*}}{\partial \chi}=\frac{1}{\chi}\left(\frac{V^{*}}{U^{*}}-\zeta^{*}\right)
$$

where $U^{*}$ and $V^{*}$ are the mean streamwise and wall-normal velocities at the interface. The wall $(y=0)$ and free-stream $(y \rightarrow \infty)$ boundary conditions on $F$ are,

$$
F_{B}(0)=0 ; \quad \frac{d F_{B}}{d \zeta}(0)=0 ; \quad \frac{d F_{T}}{d \zeta}(y \rightarrow \infty)=1 .
$$




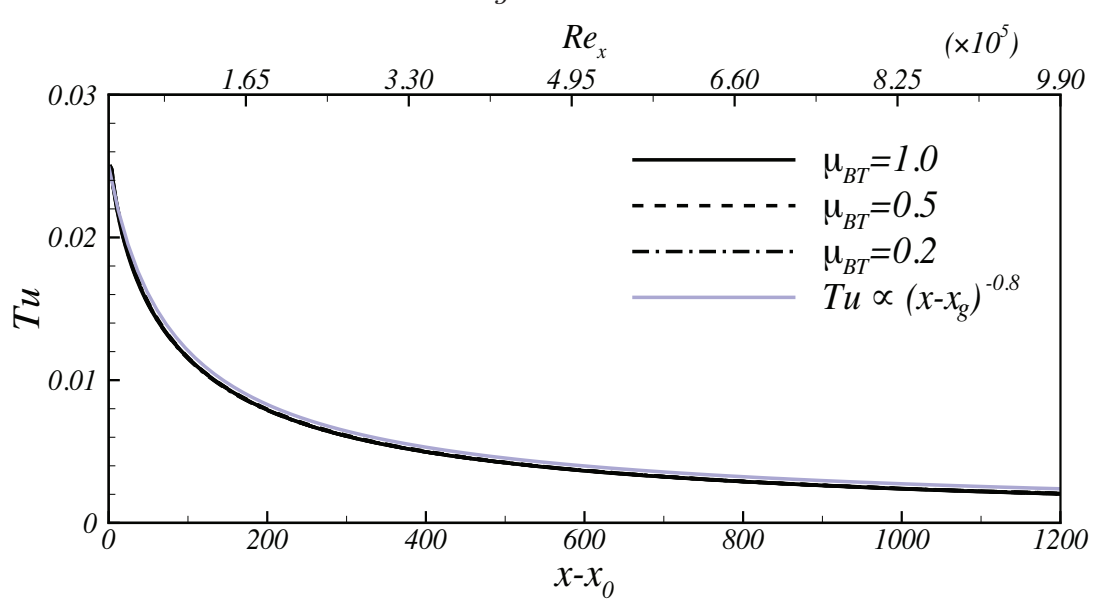

FiguRE 2. Streamwise decay of the free-stream turbulence intensity. At the inlet, $T u=2.5 \%$.

In addition, at the interface location, $\zeta=\zeta^{*}$, the following velocity and stress continuity conditions must be satisfied,

$$
F_{B}=F_{T} ; \quad \frac{d F_{B}}{d \zeta}=\frac{d F_{T}}{d \zeta} ; \quad \mu_{B} \frac{d^{2} F_{B}}{d \zeta^{2}}=\mu_{T} \frac{d^{2} F_{T}}{d \zeta^{2}} .
$$

Equations (2.12-2.15) do not admit a similarity solution. Therefore, obtaining a meanvelocity profile to apply as an inflow condition in the DNS requires marching the above equations from an upstream location up to the DNS inflow plane, $x_{0}$. Two additional requirements are imposed: (i) All the two-fluid DNS must start at the same distance $x_{0}$ from the leading edge and (ii) the film thickness must be $10 \%$ at that location. Since both the boundary layer and the film spread at different rates, an iterative procedure is required to satisfy these requirements. Equations (2.12-2.15) are solved starting upstream of $x_{0}$ with a guess of the film thickness at that location (the guess is larger than $10 \%$ relative to the local boundary-layer thickness since the film spreading rate is slower than the outer flow). The solution is marched downstream up to the inlet of the DNS domain, and the film thickness is verified at $x_{0}$. This procedure is iterated to convergence, and ensures that the inflow profiles for the single- and two-fluid DNS are solutions to the boundary-layer equations, start at the same distance to the leading edge, and have the desired film thickness.

In order to prescribe realistic free-stream vortical disturbances, isotropic turbulence was synthesized at the inlet. Following the work by Jacobs \& Durbin (2001), the disturbances are expressed in terms of Fourier modes in the spanwise dimension and in time, and a superposition of OS and Squire eigenfunctions in the wall-normal direction. The eigenfunctions are solutions to the homogeneous OS and Squire equations for the two-fluid boundary-layer. The free-stream turbulence satisfies the von Kármán spectrum

$$
E(\kappa)=T u^{2} \frac{L^{5} \kappa^{4}}{C\left[1+(\kappa L)^{2}\right]^{17 / 6}},
$$

where $C=0.688 ; \kappa \equiv \sqrt{\omega^{2}+k_{y}^{2}+k_{z}^{2}}$ and $L$ are the wavenumber and turbulence length scale, respectively. Here, the wall-normal and spanwise wavenumbers are denoted $k_{y}$ and $k_{z}$, respectively, and $\omega$ is the frequency of the perturbation. Note that $L \equiv(55 C / 9 \pi) L_{11}$ 


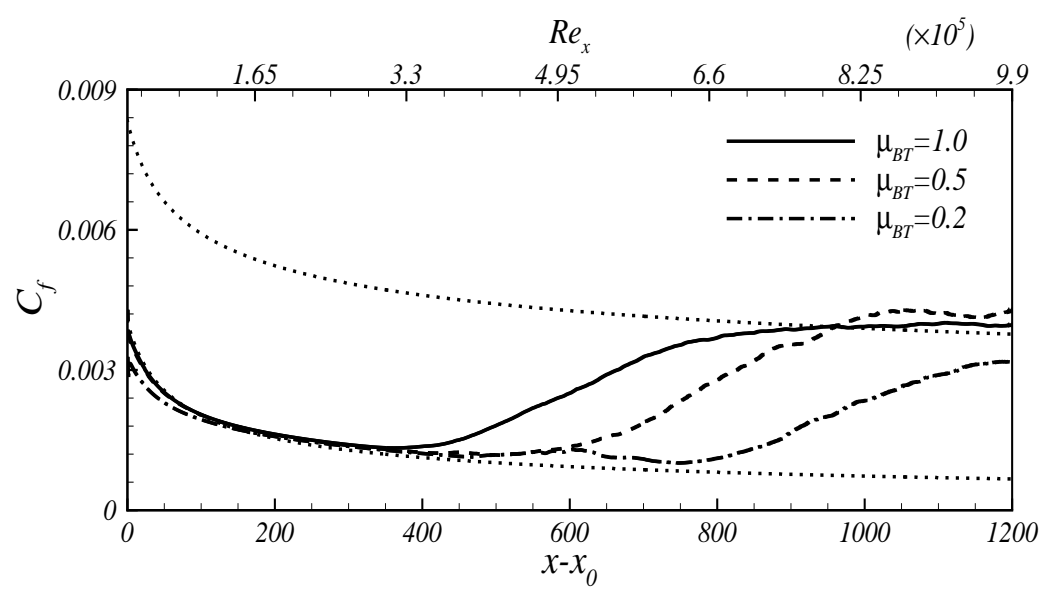

Figure 3. Downstream evolution of the skin friction coefficient for $T u=2.5 \%$.

and $L_{11}=\int_{0}^{\infty} \overline{u^{\prime}(x) u^{\prime}(x+r)} d r / T u^{2}$. The turbulence intensity, $T u$, is given by

$$
T u=\sqrt{\frac{2}{3} \int_{0}^{\infty} E(\kappa) d \kappa .}
$$

Two turbulence intensities $(T u=2.5 \%$ and $3.5 \%)$ were examined in the present study. In the case with $T u=2.5 \%$, the initial turbulence intensity decays to $0.2 \%$ near the exit of the simulation domain as shown in figure 2 . The decay rate of the turbulence intensity has the form, $T u \propto\left(x-x_{g}\right)^{-n}$ where $x_{g}$ is the virtual origin of the grid turbulence and $n \simeq 0.8$. This exponent is consistent with the experimental results by Kurian \& Fransson (2009), where $T u^{2}$ is shown to decay according to an exponential rate in the range, $-1.6<2 n<-1.2$.

\section{Reynolds-averaged statistics}

In this section, various statistical measures related to bypass transition in two-fluid boundary layers are presented, and the discussion is focused on the lower turbulence intensity simulations $(T u=2.5 \%)$. The mechanism underlying the observed trends is further analyzed in $\S 4$ by examining instantaneous and conditionally-sampled flow fields. Finally, $\S 5$ reports on the efficacy of the wall film in the case of higher $T u$.

In order to provide clear evidence of transition delay in the two-fluid configuration, the skin-friction coefficient, $C_{f}$, is reported in figure 3 . Transition to turbulence causes a substantial increase in $C_{f}$, and it is evident from figure 3 that a less viscous wall-film suppresses the onset of transition. The skin friction curve departs significantly from the Blasius value $\left(C_{f}=0.664 / \sqrt{R e_{x}}\right)$ starting at $x-x_{0} \approx 400,500$ and 750 when $\mu_{B T}=1.0$, 0.5 and 0.2 , respectively. The completion of transition is herein defined as the point where $C_{f}$ attains its maximum near the turbulent correlation $\left(C_{f}=0.455 /\left[\ln \left(0.06 R e_{x}\right)\right]^{2}\right)$. Transition is complete at $x-x_{0} \approx 1100$ and 1000 for $\mu_{B T}=1.0$ and 0.5 , respectively. In the case of $\mu_{B T}=0.2$, the fully-turbulent state is not reached within the computational domain.

A slightly more detailed view of the changes to $C_{f}$ in the case of transition delay is sought. A modification of the identity by Fukagata, Iwamoto \& Kasagi (2002), referred to hereafter as FIK, is adopted. The FIK identity is computed from the triple integration of the Reynolds-averaged Navier-Stokes (RANS) equations for the streamwise momentum in 
the wall normal direction, and it can provide a detailed view of the various contributions to $C_{f}$. For the present flow, the FIK identity is modified to take into account the viscosity distribution,

$$
\begin{aligned}
C_{f, F I K} & =\underbrace{\frac{4}{\delta_{99}^{2} R e_{\delta_{0}}} \int_{0}^{\delta_{99}} \bar{\nu} U \mathrm{~d} y}_{F I K_{\text {lam }}}-\underbrace{\frac{4}{\delta_{99}^{2}} \int_{0}^{\delta_{99}}\left(\delta_{99}-y\right) \overline{u^{\prime} v^{\prime}}}_{F I K_{\overline{u^{\prime} v^{\prime}}}} \mathrm{d} y \\
& +\underbrace{\frac{4}{\delta_{99}^{2} R e_{\delta_{0}}} \int_{0}^{\delta_{99}}\left(\delta_{99}-y\right)\left(-U \frac{\partial \bar{\nu}}{\partial y}+\overline{\nu^{\prime} \frac{\partial u^{\prime}}{\partial y}}\right) \mathrm{d} y}_{F I K_{v i s}} \\
& -\frac{4}{\delta_{99}^{2}} \int_{0}^{\delta_{99}}\left(\delta_{99}-y\right) U V \mathrm{~d} y \\
& -\frac{2}{\delta_{99}^{2}} \int_{0}^{\delta_{99}}\left(\delta_{99}-y\right)^{2}\left[\frac{\partial}{\partial x}(U U)+\frac{\partial}{\partial x}\left(\overline{u^{\prime} u^{\prime}}\right)\right] \mathrm{d} y \\
& +\frac{2}{\delta_{99}^{2} R e_{\delta_{0}}} \int_{0}^{\delta_{99}}\left(\delta_{99}-y\right)^{2}\left[\frac{\partial}{\partial x}\left(\bar{\nu} \frac{\partial U}{\partial x}\right)+\frac{\partial}{\partial x}\left(\overline{\nu^{\prime} \frac{\partial u^{\prime}}{\partial x}}\right)\right] \mathrm{d} y \\
& +\frac{2}{\delta_{99}^{2}} \int_{0}^{\delta_{99}}\left(\delta_{99}-y\right)^{2}\left(-\frac{\partial P}{\partial x}\right) \mathrm{d} y .
\end{aligned}
$$

Here, FIK lam,$F I K \overline{u^{\prime} v^{\prime}}$, FIK vis denote the contributions of the laminar base flow, Reynolds shear stress and viscosity variation in the $y$ direction, respectively. Note that the overbar represents time-averaged quantities. In equation (3.1), $C_{f}$ is decomposed into the effects of mean and fluctuating motion as well as the variable local viscosity. Generally, skin-friction is related to the near-wall turbulence structures (Kravchenko, Choi \& Moin 1993; Choi, Moin \& Kim 1994). This formulation demonstrates the higher impact of near-wall turbulence motions as dictated by the moments in the integrals.

Figure 4 displays contributions of the first three terms of equation 3.1 to the skinfriction coefficient for $\mu_{B T}=1.0$ and 0.2 . The sum of the remaining terms is also included and is denoted FIK other. The skin-friction coefficient obtained from the FIK identity $\left(C_{f, F I K}\right)$ is in good agreement with the time-averaged $C_{f}$ evaluated during the DNS simulation.

In the pre-transitional boundary layer, the total $C_{f}$ shadows the laminar curve, with an offset due to the energetic streak distortions. While the streaks have a significant $\overline{u^{\prime} u^{\prime}}$ contribution, they do not have an appreciable associated Reynolds shear stress. The term $F I K \overline{u^{\prime} v^{\prime}}$ is therefore relatively small in the pre-transitional regime. With the onset of transition to turbulence, energy is re-distributed from $\overline{u^{\prime} u^{\prime}}$ to $\overline{u^{\prime} v^{\prime}}$ and the increase in $F I K \overline{u^{\prime} v^{\prime}}$ is mirrored in the total $C_{f}$ curve. Relative to the single-fluid flow, the abrupt increase of $F I K \overline{u^{\prime} v^{\prime}}$ is delayed downstream in the presence of the wall film.

The large contribution of FIK lam seen in figure 4(b) near the inlet $\left(x-x_{0}<100\right)$ is due to the fuller profile of the streamwise mean velocity. The change of viscosity in the wall-normal direction leads to the decrease in $C_{f}$ as demonstrated by the figure. This is mainly due to the large positive gradient of mean viscosity. Note that, unlike the instantaneous viscosity profile which is sharp, the average viscosity has a smoother profile due to the time dependence of the interface location. Nonetheless $\bar{\nu}(y)$ retains a sufficiently large $\partial \bar{\nu} / \partial y$ up to $x-x_{0} \approx 200$.

The downstream growth of boundary-layer $99 \%$ and momentum thicknesses are shown in figure $5 a$ and $c$. Initially $\delta_{99}$ and $\theta$ increase gradually following the Blasius solution 


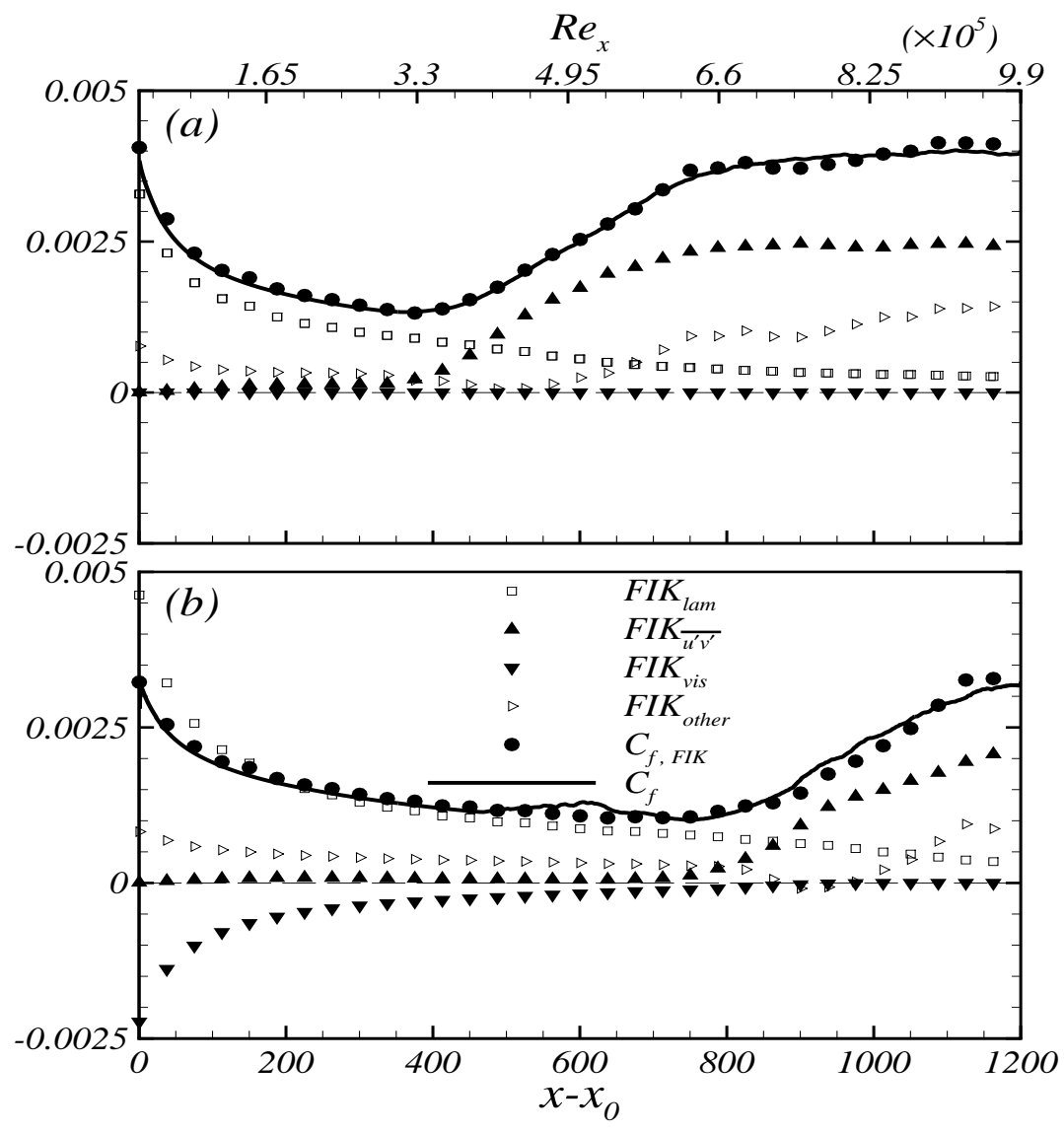

FiguRE 4. Contributions to the Skin friction coefficients for $(a) \mu_{B T}=1.0$ and $(b) \mu_{B T}=0.2$.

in the laminar region, but subsequently increase at a higher rate in the transition zone. The rise in the momentum thickness reflects the sharp increase in skin friction across the transition region, and is delayed further downstream in the presence of the wall-film. The downstream spreading of the wall film is shown in figure $5 b$. Its growth rate is slower than that of $\delta_{99}$ and, as a result, the local ratio of the film-to-boundary-layer thickness, $d / \delta_{99}$, decreases downstream. The film thicknesses for $\mu_{B T}=0.5$ and 0.2 are only reported up to $x-x_{0} \approx 450$ and 600 , respectively. In the transition zone, the wall film is detached due to vigorous buffeting by the turbulence spots.

The influence of the viscosity ratio on the mean velocity at several $x$ locations is shown in figure 6 . Here, the $y$-axis is normalized by the local boundary layer thickness $\delta_{99}$. The purpose of introducing the wall film is achieved as the mean shear in the pre-transitional regime is increasingly contained within the film at lower film viscosities. Upstream of transition, the fuller mean profile within the less viscous film flow explains the large contribution of FIK lam to $C_{f}$ shown in figure 4(b). After the onset of transition, the mean velocity in the single-fluid boundary layer increases near the wall. However, the mean velocity for $\mu_{B T}=0.2$ retains the laminar profile up to $x-x_{0}=800$ due to transition delay. With weaker outer shear in the two-fluid flow, it is anticipated that the lift-up mechanism in the bulk fluid will be weakened, although the change in the disturbance field within the film is more difficult to anticipate. 

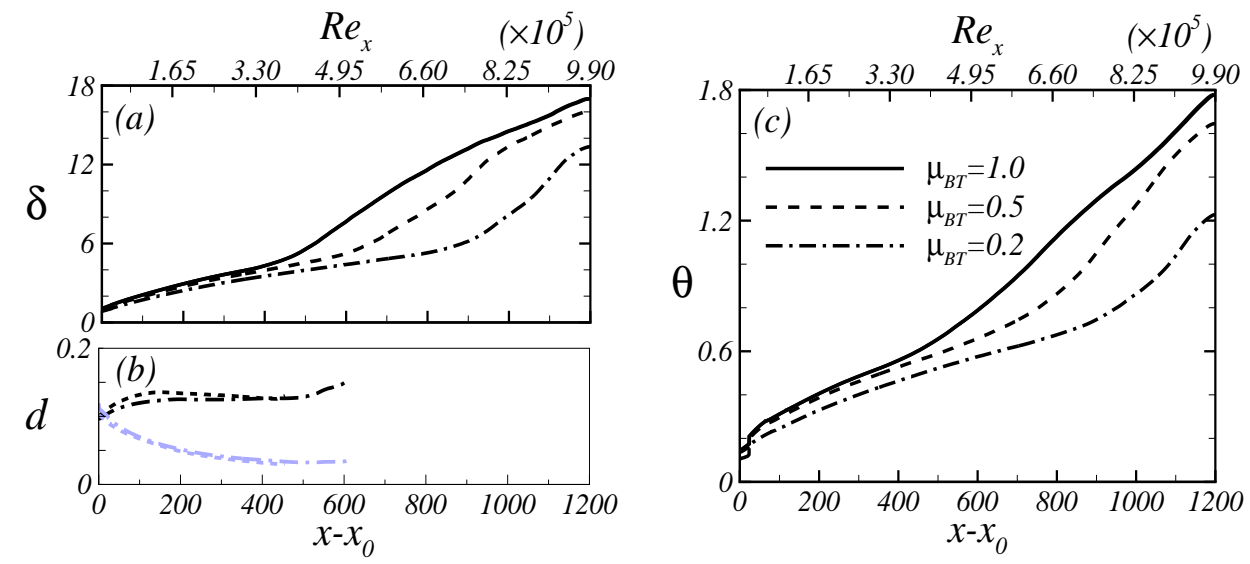

Figure 5. Profiles of $(a)$ boundary layer $99 \%$ thicknesses $\left(\delta_{99} / \delta_{0}\right),(b)$ film thicknesses $\left(d / \delta_{0}\right.$ : black lines, $d / \delta_{99}$ : light lines) and $(c)$ momentum thicknesses $\left(\theta / \delta_{0}\right)$.

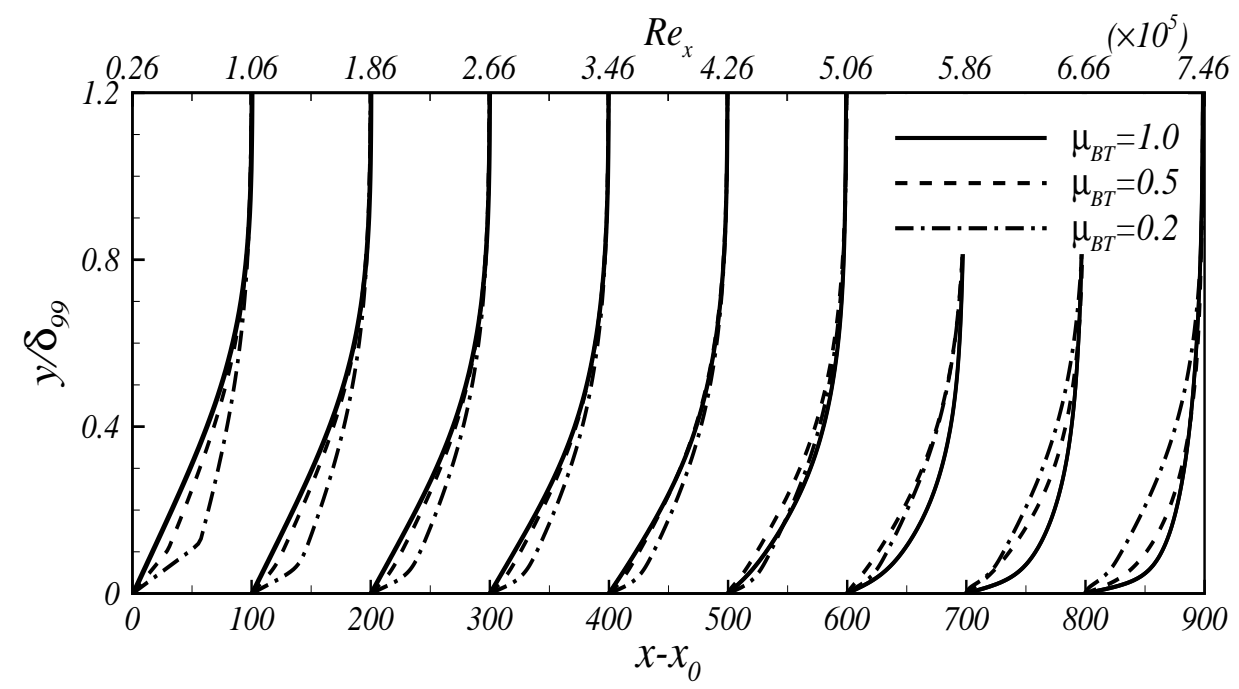

FiguRE 6. Mean velocity profiles at various downstream locations. On the $x$-axis, the distance between major tick marks denotes $U_{x}=1.0 U_{\infty}$.

The root-mean-square (rms) of the streamwise velocity fluctuations is plotted in figure 7 for different $x$-locations. The streamwise velocity fluctuations for $\mu_{B T}=1.0$ amplify with downstream distance. The distribution of $u_{r m s}^{\prime}$ shows a maximum approximately in the middle of the laminar boundary layer $\left(y / \delta_{99} \approx 0.4\right.$ corresponding to $\left.y / \delta^{*} \approx 1.2\right)$, which is in agreement with previous results from experiments and numerical simulations (Matsubara \& Alfredsson 2001; Brandt, Schlatter \& Henningson 2004). The laminar $u_{r m s}^{\prime}$ profiles of $\mu_{B T}=1.0\left(x-x_{0}<300\right)$ have the appearance of Klebanoff modes. After the onset of transition, the peak of the velocity fluctuation shifts towards the wall.

The general trend of $u_{r m s}^{\prime}$ described above is also observed in the two-fluid flows, although with two important changes. First, the amplitude of fluctuations becomes weaker with decreasing film viscosity. This is consistent with the linear prediction that lower viscosity wall films attenuate the energy amplification inside the boundary layer (Saha, Jung 


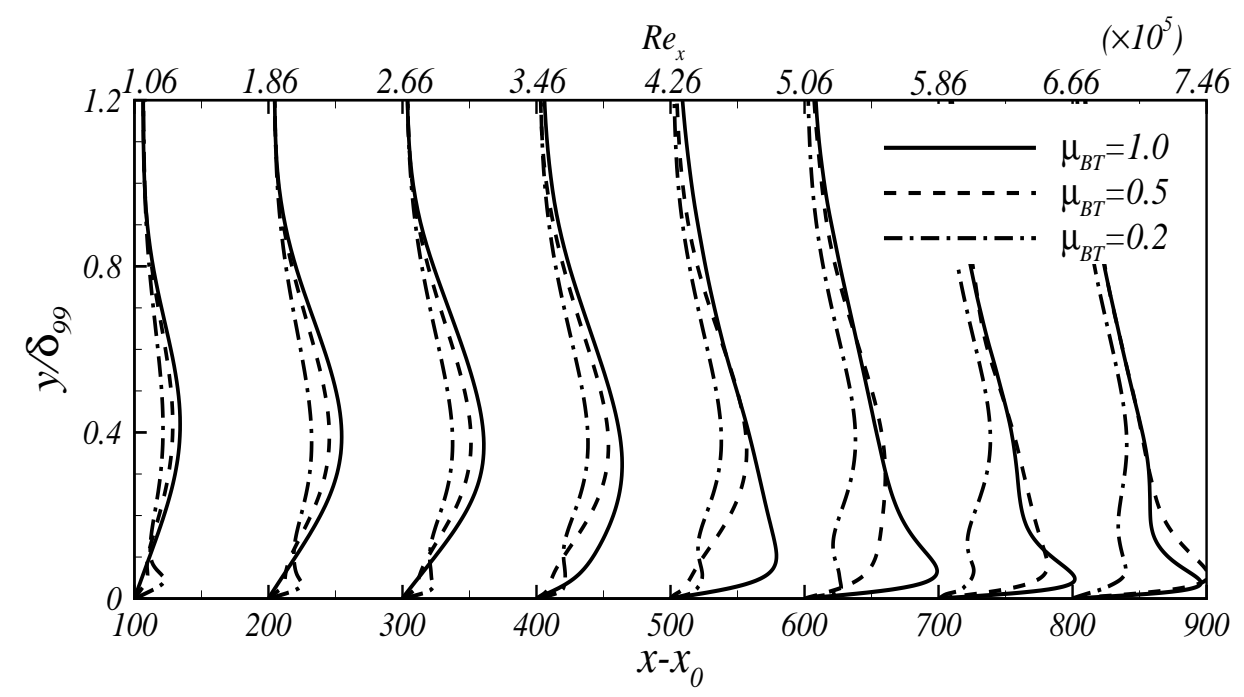

FiguRE 7. Profiles of the root-mean-square streamwise velocity fluctuations at various downstream locations. On the $x$-axis, the distance between major tick marks denotes $u_{r m s}^{\prime}=0.17 U_{\infty}$.

\& Zaki 2010). Second, a new peak in $u_{r m s}^{\prime}$ emerges near the interface $\left(y / \delta_{99} \approx 0.05\right)$ in the laminar region in the case of $\mu_{B T}=0.2$. This peak persists up to the pre-transitional regime. Both observations will be quantified, and will be explained with the aid of linear theory.

The maximum value of $u_{r m s}^{\prime}$ is extracted at every downstream location and is plotted in figure 8. Here, the outer peak refers to the maximum located in the middle of boundary layer and the inner peak denotes the one closer to the wall. In the single-fluid boundary layer, only one peak exists and is reproduced in both figures for reference. The amplification of the outer peak in the laminar region $\left(x-x_{0}<200\right)$ is reduced with decreasing film viscosity. This suggests that the streak amplification is weakened in two-fluid boundary layers. After transition onset, the outer peaks of $\mu_{B T}=1.0$ and 0.5 grow rapidly $\left(x-x_{0}=400\right.$ and 500) and attain maxima at $x-x_{0}=640$ and 800 , respectively. It is seen in figure 8 (b) that the inner peak of $\mu_{B T}=0.5$ is only discernible in the region of $x-x_{0}<400$.

The outer peak is weakest for $\mu_{B T}=0.2$. In contrast to higher viscosity ratios, the inner peak has a comparable amplitude to the outer streaks when $\mu_{B T}=0.2$. Beyond $x-x_{0}>800$, the outer and inner peaks in $u_{r m s}^{\prime}$ are indistinguishable. They increase sharply and reach a peak value at $x-x_{0}=1,080$.

In figure 9 , the wall-normal locations of the maximum $u_{r m s}^{\prime}$ are displayed. The laminar outer peaks lie between $0.38<y / \delta_{99}<0.5$ as shown in figure 7 . It is clear that the outer peaks move rapidly towards the wall in the transition regime. In the fully-turbulent regime, when $\mu_{B T}=1.0$ or 0.5 , the outer peak is located at $y / \delta_{99} \approx 0.025$. This $y$ location corresponds to $y^{+} \approx 14.5$ which is similar to the value from previous experiments (e.g. Matsubara \& Alfredsson 2001). The inner peak for two-fluid boundary layers is placed between $0.03<y / \delta_{99}<0.1$.

The Reynolds-averaged streamwise velocity fluctuations reveal that the laminar streaks are weakened in two-fluid boundary layers. The origin of this trend can be explained with the aid of linear theory. The linear perturbation equations for the wall-normal velocity 

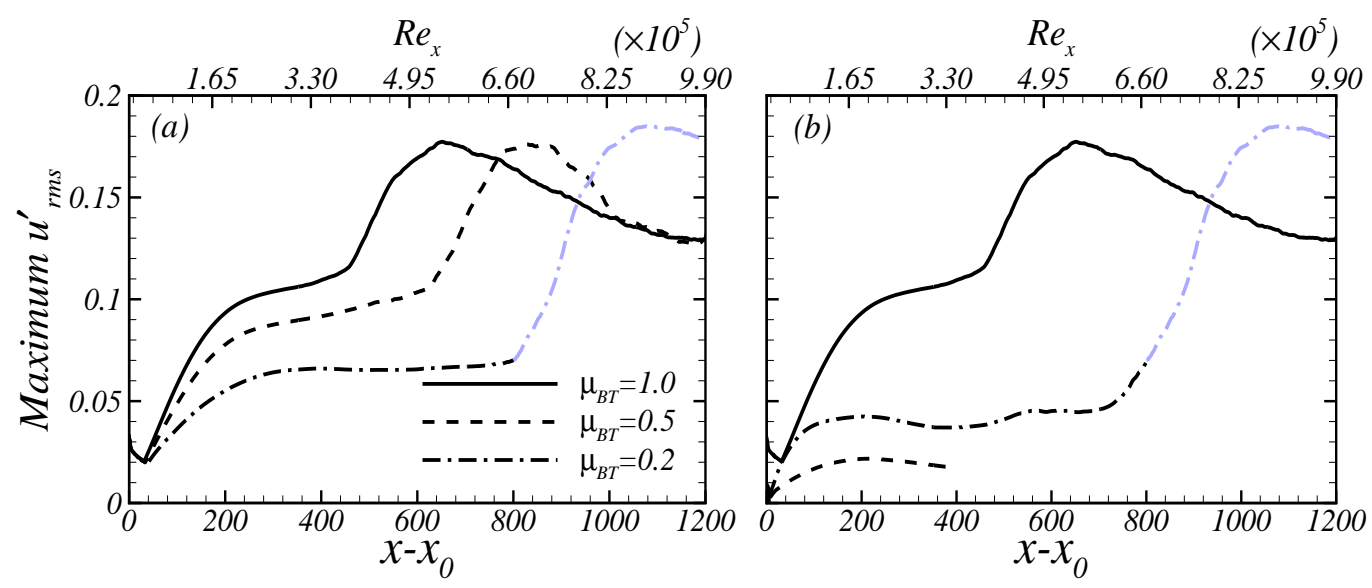

Figure 8. Maximum $u_{r m s}^{\prime}$ versus downstream distance for $(a)$ outer peak and $(b)$ inner peak. For $\mu_{B T}=0.2$, the two peaks become indistinguishable in the region $x-x_{0}>800$ (light line).

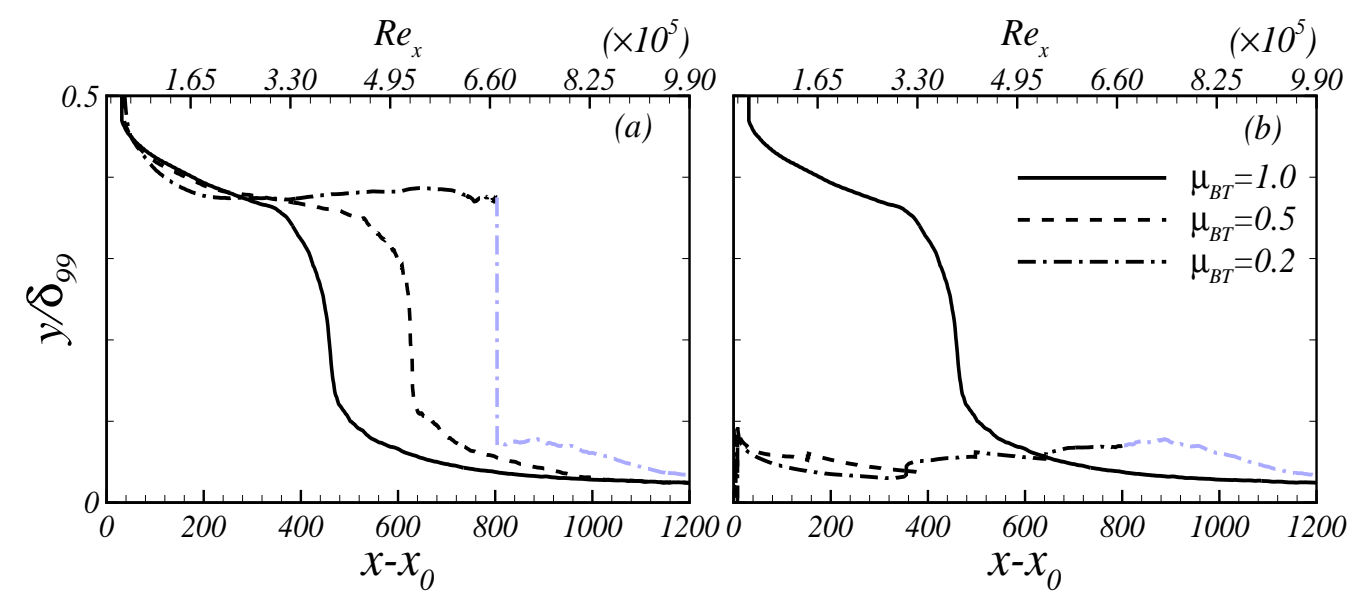

FiguRE 9. Wall normal location of maximum $u_{r m s}^{\prime}$ versus downstream distance for $(a)$ outer peak and $(b)$ inner peak. For $\mu_{B T}=0.2$, the outer peak moves towards the wall quickly in the transition zone, and the two peaks become indistinguishable downstream (light line).

and vorticity are,

$$
\frac{\partial}{\partial t}\left[\begin{array}{c}
\nabla^{2} v_{j}^{\prime} \\
f^{\prime} \\
\eta_{j}^{\prime}
\end{array}\right]=\left[\begin{array}{ccc}
\frac{d^{2} U_{j}}{d y^{2}} \frac{\partial}{\partial x}+\nu_{j} \nabla^{4}-U_{j} \frac{\partial}{\partial x} \nabla^{2} & 0 & 0 \\
\delta(y-d) & -U(d) \frac{\partial}{\partial x} & 0 \\
\frac{d U_{j}}{d y} \frac{\partial}{\partial z} & 0 & \nu_{j} \nabla^{2} \eta-U_{j} \frac{\partial}{\partial x}
\end{array}\right]\left[\begin{array}{c}
v_{j}^{\prime} \\
\mathrm{f}^{\prime} \\
\eta_{j}^{\prime}
\end{array}\right]
$$

where $\delta(y-d)$ is the delta function at the interface location and $\mathrm{f}^{\prime}$ is the interfacial displacement. These equations have appeared previously in the literature on linear stability of two-fluid flows, albeit they are often reported in Fourier space (e.g. Yih 1967; Yecko \& Zaleski 2005; Malik \& Hooper 2007; Cheung \& Zaki 2010). No-slip and no-penetration boundary conditions are applied at the wall. Homogeneous boundary conditions are im- 
posed on $v^{\prime}, \frac{\partial v^{\prime}}{\partial y}$ and $\eta^{\prime}$ in the free-stream.

$$
\begin{aligned}
v_{B}^{\prime}(0) & =0 ; \quad \partial_{y} v_{B}^{\prime}(0)=0 \quad ; \eta_{B}^{\prime}(0)=0 \\
v_{T}^{\prime}(y \rightarrow \infty) & =0 ; \partial_{y} v_{T}^{\prime}(y \rightarrow \infty)=0 ; \eta_{T}^{\prime}(y \rightarrow \infty)=0
\end{aligned}
$$

In addition, the perturbation field must satisfy continuity of velocity and stresses linearized to the mean interface location, which can be expressed in terms of $v$ and $\eta$,

$$
\begin{gathered}
{\left[v^{\prime}\right]=0 ;} \\
{\left[\frac{\partial v^{\prime}}{\partial y}\right]=\left[\frac{d U}{d y}\right] \frac{\partial f^{\prime}}{\partial x} ;} \\
{\left[\mu\left(\frac{\partial^{2} v^{\prime}}{\partial y^{2}}-\nabla_{x z}^{2} v^{\prime}\right)\right]=\left[\mu \frac{d^{2} U}{d y^{2}}\right] \frac{\partial f^{\prime}}{\partial x} ;} \\
{\left[\rho\left(\frac{\partial}{\partial t} \frac{\partial v^{\prime}}{\partial y}+U \frac{\partial^{2} v^{\prime}}{\partial x \partial y}-\frac{d U}{d y} \frac{\partial v^{\prime}}{\partial x}\right)-\mu \frac{\partial^{3} v^{\prime}}{\partial y^{3}}-3 \mu \nabla_{x z}^{2} \frac{\partial v^{\prime}}{\partial y}\right]=\sigma \nabla_{x z}^{4} f^{\prime} ;} \\
{\left[\eta^{\prime}\right]=-\left[\frac{d U}{d y}\right] \frac{\partial f^{\prime}}{\partial z} ;} \\
{\left[\mu \frac{\partial \eta^{\prime}}{\partial y}\right]=-\left[\mu \frac{d^{2} U}{d y^{2}}\right] \frac{\partial f^{\prime}}{\partial z} .}
\end{gathered}
$$

where $\nabla_{x z}^{2}$ is the Laplacian operator in the $x-z$ plane, [.] denotes the change across the interface, $(.)_{T}-(.)_{B}$, and $\sigma$ is the surface tension.

As seen in equation (3.2), wall-normal vorticity can be generated by tilting of the mean spanwise vorticity. In the limit of streamwise-elongated disturbances, the amplification of $\eta^{\prime}$ is representative of the behaviour of $u^{\prime}$, or the streaks, since $\eta^{\prime}=\partial u^{\prime} / \partial z-\partial w^{\prime} / \partial x$. Figure 10 shows the quantity $v_{r m s}^{\prime} d U / d y$, which is closely related to the forcing term of $\eta^{\prime}$ in equation (3.2), at four different $x$ positions. In general, this term decays downstream in the laminar-flow regime due to the decay of $v_{r m s}^{\prime}$. However, the profiles show a clear variability with viscosity ratio. In the single-fluid case, the profile has only one peak, while the two-fluid cases have two peaks: an outer one which is located at $y / \delta_{99} \approx 0.55$ and an inner one at $y / \delta_{99}=0.1$. The magnitude of the outer peak diminishes with decreasing $\mu_{B T}$ since the wall film absorbs the mean shear which becomes weaker in the outer fluid (see figure 6). This weakening of the vorticity tilting term in the top fluid explains the weaker Klebanoff streaks in figure 7. On the other hand, the inner peak is intensified with the reduction in viscosity ratio (see figure 10a). This can lead to stronger $\eta^{\prime}$, and therefore streaks, near the interface. At the interface itself, this effect is captured by the matching condition 3.4e, where the jump in mean shear can become a source of $\eta^{\prime}$ if the interface is deformed. The response is the inner peak in $u_{r m s}^{\prime}$ reported in figure $8 b$, which is enhanced as $\mu_{B T}$ is reduced.

Linear theory provides a viable starting point to explain the changes in the $u$-perturbation field in the non-linear simulations. In the context of the DNS, the relevant term is $\overline{u^{\prime} v^{\prime}}(\partial U / \partial y)$, or production due to the Reynolds' shear stress. This term is analyzed in detail below. It is also important to note that weaker $u_{r m s}^{\prime}$ does not guarantee stability. Nolan \& Zaki (2013) showed that rare high-amplitude streaks are the key sites for transition onset. Therefore, a closer examination of the population of streaks is required in order to determine whether the extreme values of the distribution of streak amplitudes are also diminished due to the wall film. 

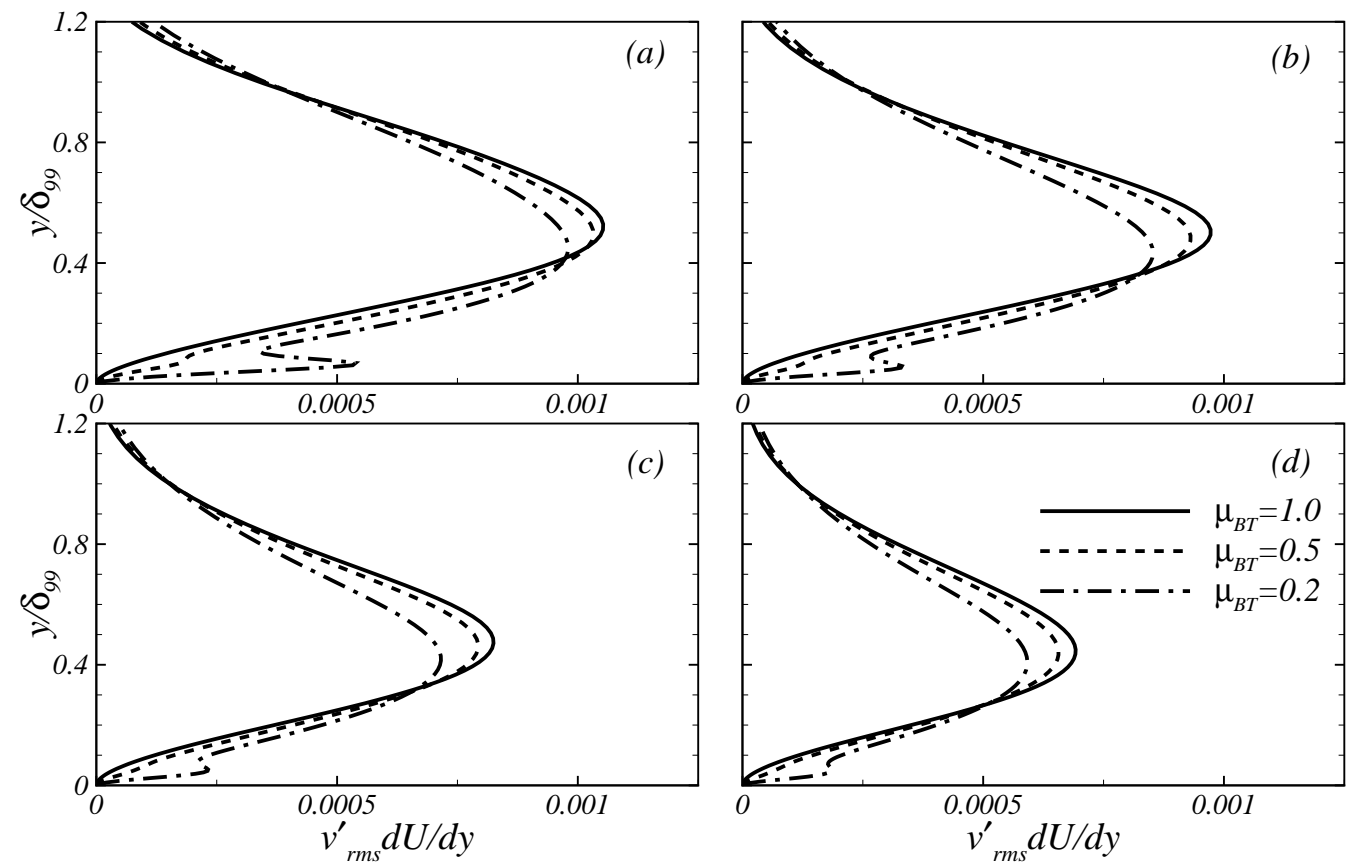

Figure 10. profiles of $v_{r m s}^{\prime} d U / d y$ for $(a) x-x_{0}=50,(b) x-x_{0}=100,(c) x-x_{0}=150$ and $(d)$ $x-x_{0}=200$.

\section{Conditionally-averaged fields and instantaneous flow structures}

A comparison of instantaneous $u^{\prime}$ contours between single- and two-fluid boundary layers is provided in figures 11 and 12 . The spanwise dimension is magnified with an aspect ratio of 5:1. The dark contours mark $u^{\prime}<0$, or low-speed streaks, and the broader bright zones are occupied by high speed streaks with $u^{\prime}>0$. In figure 11(a), a patch of turbulence is observed near $x-x_{0}=400$; its inception location is situated on a low-speed streak. As the turbulent spot travels downstream, it spreads in the horizontal plane. The front of the patch propagates more rapidly than the rear, and it merges with the fully turbulent flow downstream. The amalgamation of these spots sustains the fully-turbulent region of the flow within the computational domain. This process is also described in the work by Jacobs \& Durbin (2001).

The formation of the turbulent spot takes place further downstream when $\mu_{B T}=0.2$. The irregular edge of turbulent motion is seen at $x-x_{0}=650$ in figure 12 (a). The convective speed of the spot is similar to that in the single-fluid flow. The delay of turbulent spot formation provides clear evidence that transition is delayed by the wall film. Based on previous studies, spot inception is correlated with streak amplitude (Nolan \& Zaki 2013). Therefore, the lowered streak amplitudes in the pre-transitional two-fluid boundary layer can be directly responsible for the delayed breakdown.

The top views of the perturbation fields are complemented by side views of the instantaneous flow in figures 13 and 14. The light and dark contours denote the positive and negative wall-normal velocity fluctuations, respectively. The $v$-perturbations are displayed in conjunction with the vectors. Note that the time interval, $\Delta T$, between consecutive frames in the side views (figures 13 and 14) is shorter than in the top views (figures 11 and 12) in order to capture the evolution of the secondary instability. In the singlefluid case, irregular velocity patterns are clearly visible near the edge of the boundary 

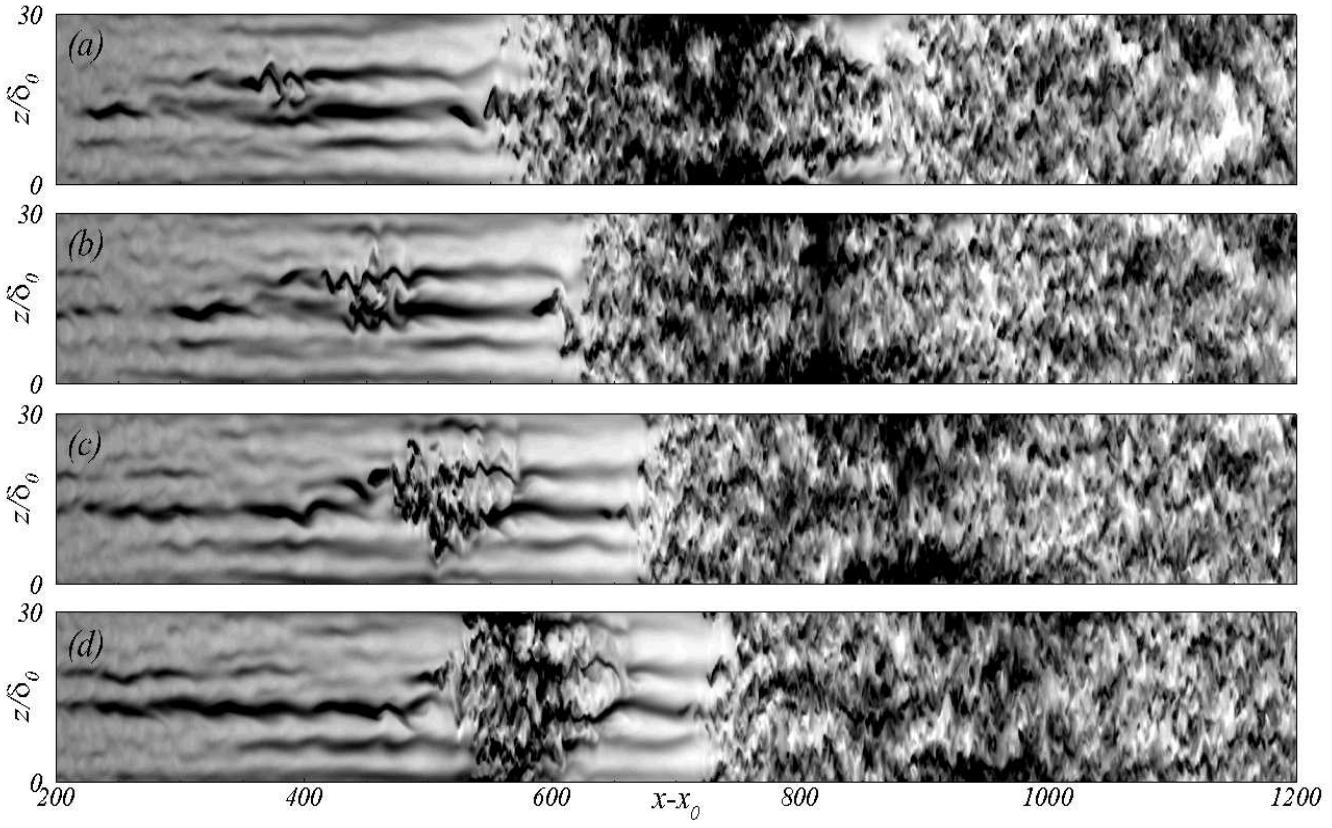

Figure 11. Instantaneous $u^{\prime}$ contours $\left(-0.15 U_{\infty}<u^{\prime}<0.15 U_{\infty}\right)$ in the $(x, z)$-plane for $\mu_{B T}=1.0$ at $(a) T_{0},(b) T_{0}+\Delta T$, (c) $T_{0}+2 \Delta T$ and $(d) T_{0}+3 \Delta T$. Here, $T_{0}$ is the reference time and $\Delta T=96 \delta_{0} / U_{\infty}$.
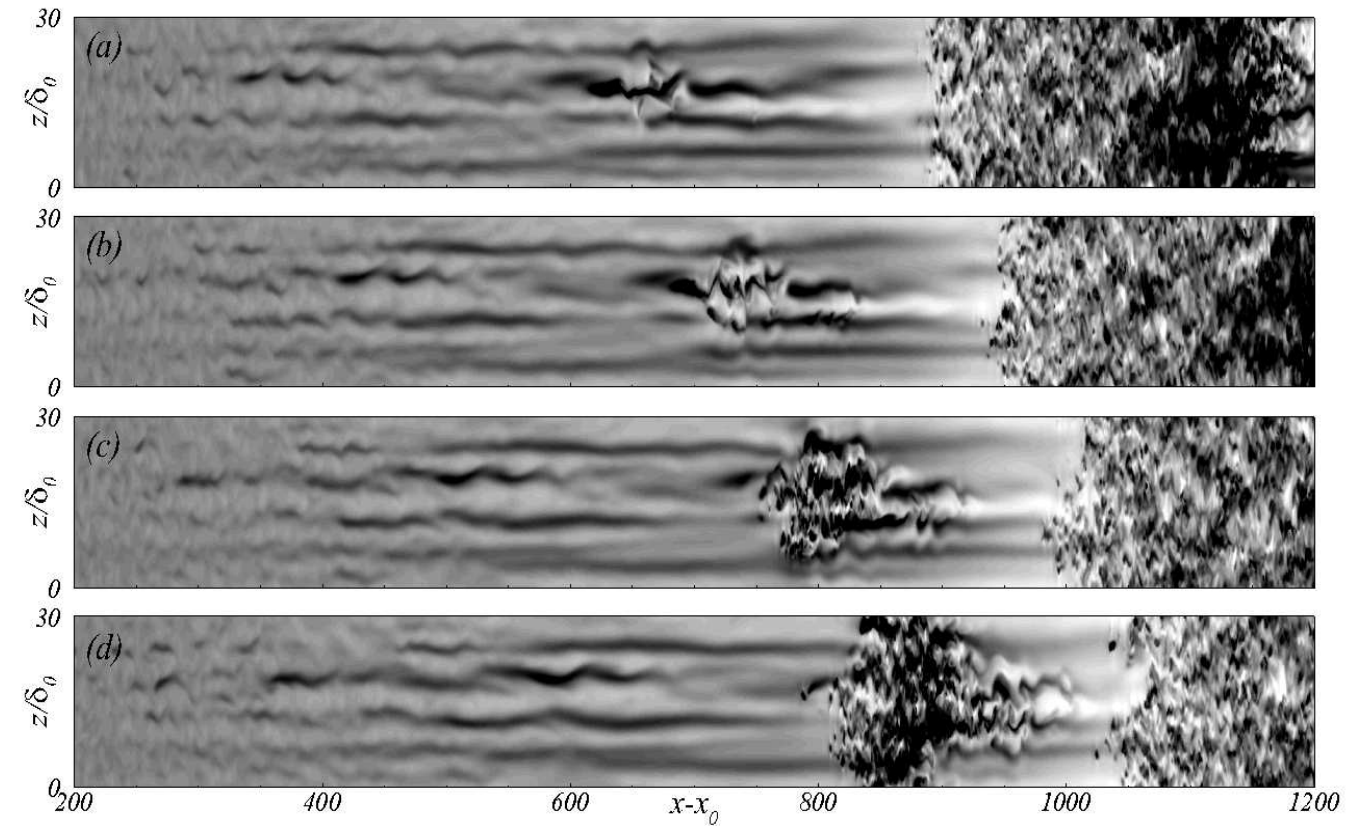

Figure 12. Instantaneous $u^{\prime}$ contours $\left(-0.15 U_{\infty}<u^{\prime}<0.15 U_{\infty}\right)$ in the $(x, z)$-plane for $\mu_{B T}=0.2$ at $(a) T_{0},(b) T_{0}+\Delta T,(c) T_{0}+2 \Delta T$ and $(d) T_{0}+3 \Delta T$. Here, $T_{0}$ is the reference time and $\Delta T=96 \delta_{0} / U_{\infty}$. 


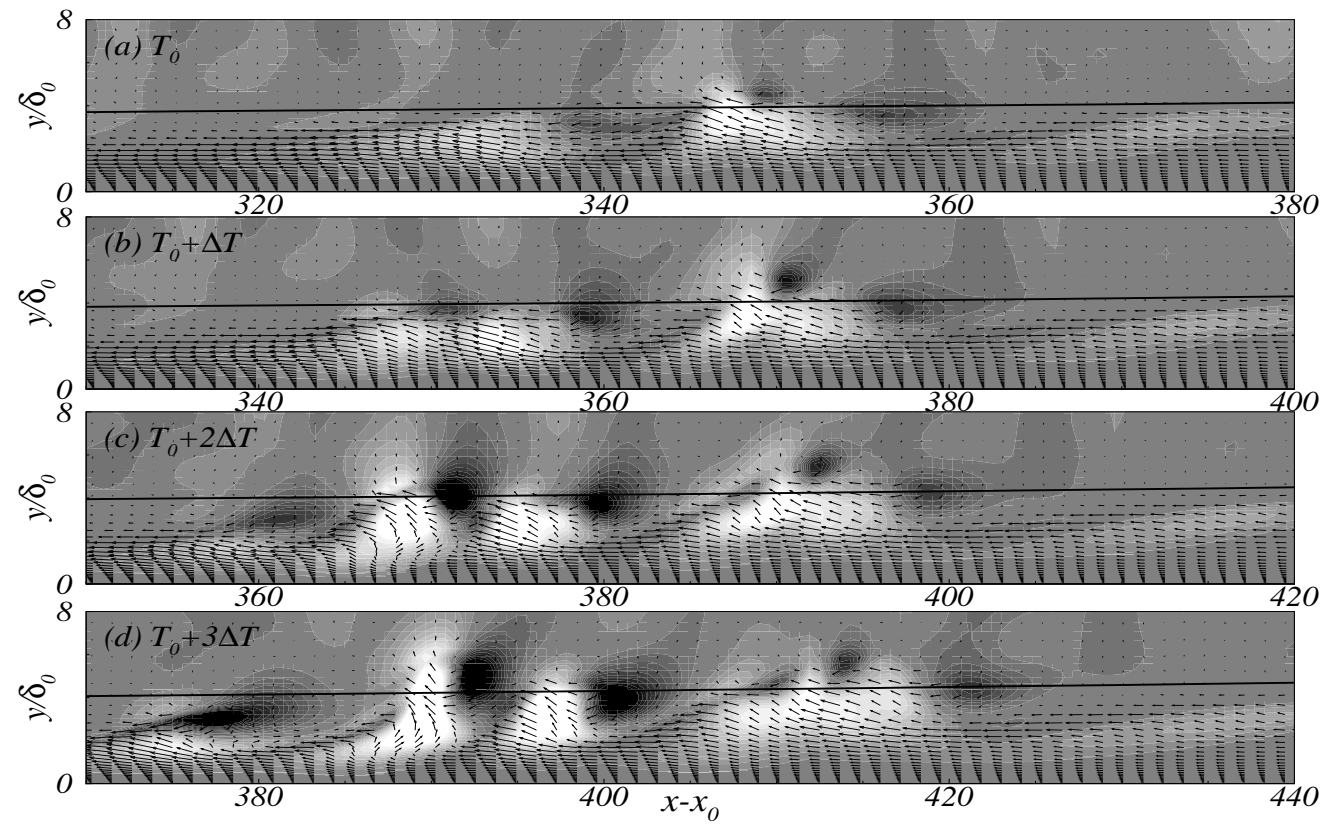

FiguRE 13. Instantaneous $v^{\prime}$ contours $\left(-0.05 U_{\infty}<v^{\prime}<0.05 U_{\infty}\right)$ in the $(x, y)$-plane for $\mu_{B T}=1.0$. Time sequence showing the evolution of the spot precursor via vectors. Black line denotes the location of the boundary-layer edge. The viewing window moves at $0.83 U_{\infty}$. Here, $\Delta T=24 \delta_{0} / U_{\infty}$
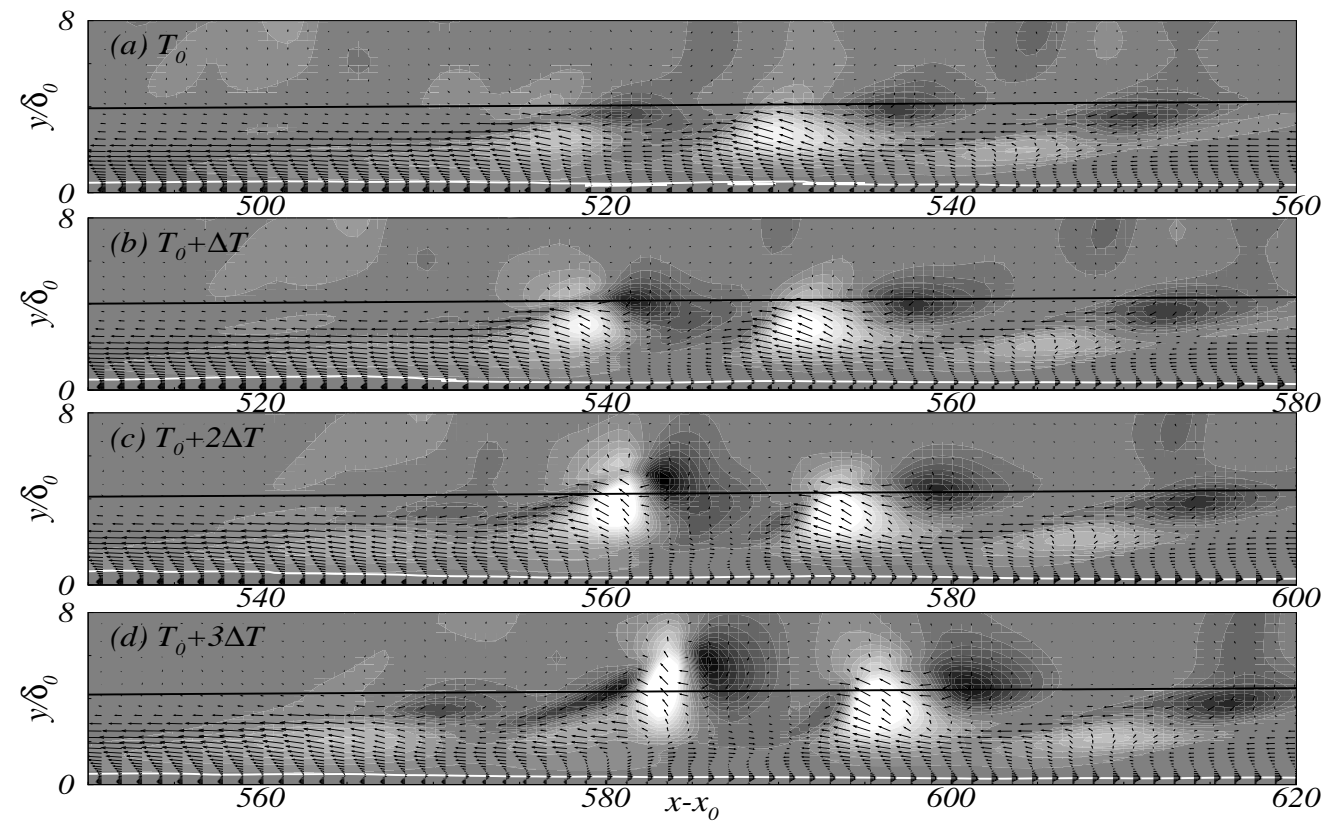

FiguRE 14. Instantaneous $v^{\prime}$ contours $\left(-0.05 U_{\infty}<v^{\prime}<0.05 U_{\infty}\right)$ in the $(x, y)$-plane for $\mu_{B T}=0.2$. Time sequence showing the evolution of the spot precursor via vectors. Black and white lines denote the locations of the boundary-layer edge and the interface, respectively. The viewing window moves at $0.83 U_{\infty}$. Here, $\Delta T=24 \delta_{0} / U_{\infty}$. 
layer $(13 c$ and $d)$. These are due to the outer secondary instability of lifted low-speed streaks which precedes the inception of the turbulent spot shown in figure 11. In the case where $\mu_{B T}=0.2$, the outer and inner peaks in the $u_{r m s}^{\prime}$ profile became indistinguishable in the transition zone. Therefore, it is not clear whether breakdown to turbulence was caused by a secondary instability mechanism that affects the outer streaks or the twofluid interface. Figure 14 captures the secondary instability which causes transition, and which starts farther downstream than in the single-fluid boundary layer. The instability is atop the lifted outer streaks, which have a weaker amplitude in figure 14 relative to the single-fluid case. Therefore, the transition mechanism is not changed but rather delayed by the introduction of the wall film.

The lift-up mechanism which leads to the amplification of streaks is related to the ejection/sweep motions which contribute to the positive production of turbulence. In order to investigate the changes to the ejection/sweep events due to the wall film, a quadrant analysis for the Reynolds shear stress is performed. The analysis of the Reynolds shear stress provides detailed information on the contributions of various events occurring in the flow to the total turbulence production (Willmarth \& Lu 1972; Brodkey, Wallace \& Eckelmann 1974). The analysis divides the Reynolds shear stress into four categories according to the signs of $u^{\prime}$ and $v^{\prime}$. The first quadrant Q1 $\left(u^{\prime}>0\right.$ and $\left.v^{\prime}>0\right)$ contains outward motion of high-speed fluid; the second quadrant Q2 $\left(u^{\prime}<0\right.$ and $\left.v^{\prime}>0\right)$ contains outward motion of low-speed fluid, or "ejections"; the third quadrant Q3 $\left(u^{\prime}<0\right.$ and $\left.v^{\prime}<0\right)$ represents inward motion of low-speed fluid; and the fourth quadrant Q4 $\left(u^{\prime}>0\right.$ and $\left.v^{\prime}<0\right)$ represents in-rush of high-speed fluid, or "sweeps". Here, Q1 and Q3 events contribute to the positive Reynolds shear stress (negative production), and Q2 and Q4 events contribute to the negative Reynolds shear stress (positive production).

Figure 15(a) shows the contributions to the Reynolds shear stress from each quadrant in the pre-transitional regime. It is evident that the magnitudes of Q2 and Q4 events are decreased in the two-fluid boundary layers, consistent with the weaker production of Klebnaoff streaks. This trend becomes more pronounced at the downstream location $x-x_{0}=400$. In figure $15(\mathrm{~b})$, the peak of the Q4 events is closer to the wall in the singlefluid flow than in the two-fluid boundary layer. This change is due to the single-fluid flow reaching the starting point of transition to turbulence. The enhanced Q2 and Q4 have also been credited to increases in the skin friction as pointed out in the discussion of figure 4 . These trends in the single-fluid boundary layer do not affect the two-fluid flow since its transition location is farther downstream.

In order to evaluate the contributions of strong ejections to the upwelling of the lifted negative streaks, conditionally-averaged flow fields are examined in figures 16 and 17 . The conditional average of flow fields is estimated surrounding a Q2 event at $y / \delta_{99}=0.5$ over the $2 \mathrm{D}$ window $1 \delta_{99} \times 2 \delta_{99}$ in the cross-flow plane. The conditions for the strong Q2 event are $-u^{\prime} v^{\prime}>H u_{r m s}^{\prime} v_{r m s}^{\prime}, u^{\prime}<0$ and $v^{\prime}>0$. The threshold $H=2$ is selected, consistent with previous studies (Littell \& Eaton 1994; Kang, Choi \& Yoo 1998; Le, Coleman \& Kim 2000). The central peak in each plot, depicting a strong ejection, is flanked by two secondary peaks generated by the opposite event. Kang, Choi \& Yoo (1998) postulated that these peaks are the signature of the pair of streamwise vortices that generate the strong Reynolds-stress-producing event. The central peak in each plot represents the combined effect of both vortices, while the secondary peaks each correspond to the effect of an individual vortex.

The central peak of the two-fluid boundary layer is much weaker than that of the single-fluid flow. This is consistent with the findings in figure 15. From the quadrant analyses, suppression of the lift-up mechanism by the wall film is plausible. The strength of the secondary peaks is also reduced when $\mu_{B T}=0.2$. 

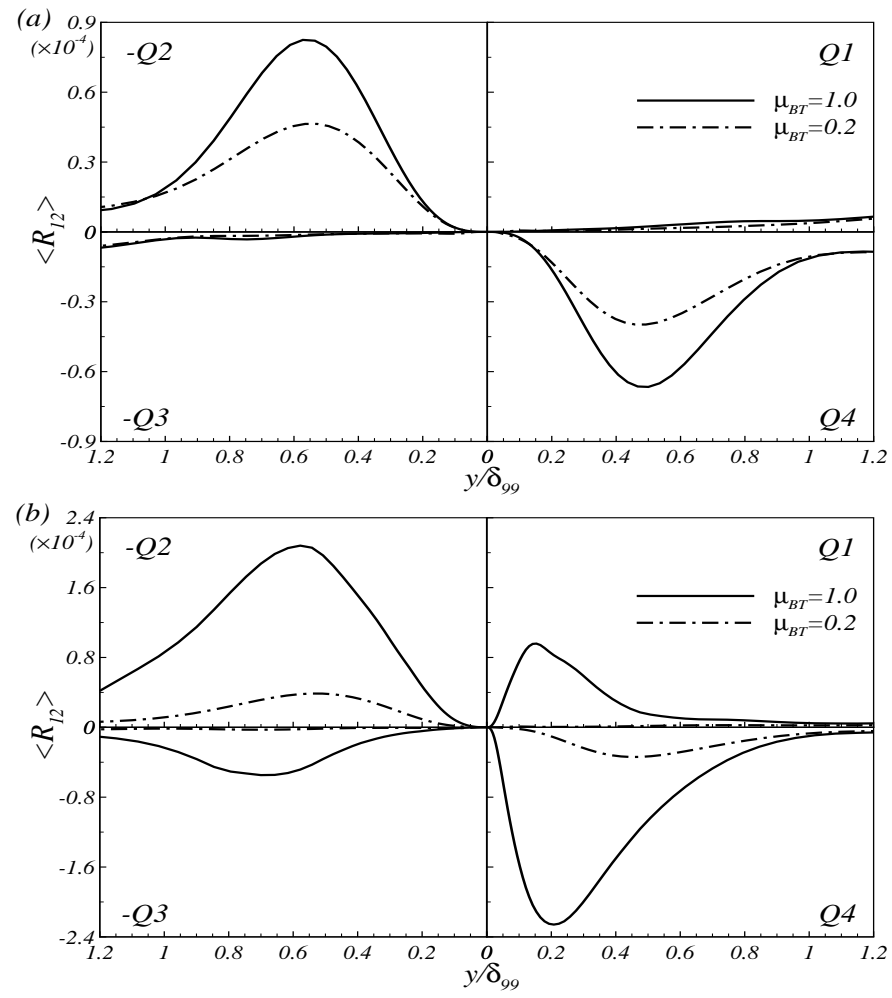

Figure 15. Contributions to the Reynolds shear stress $\left(\overline{u^{\prime} v^{\prime}}\right)$ from each quadrant at $(a)$ $x-x_{0}=200$ and $(b) x-x_{0}=400$. Each profile is normalized by $U_{\infty}^{2}$.
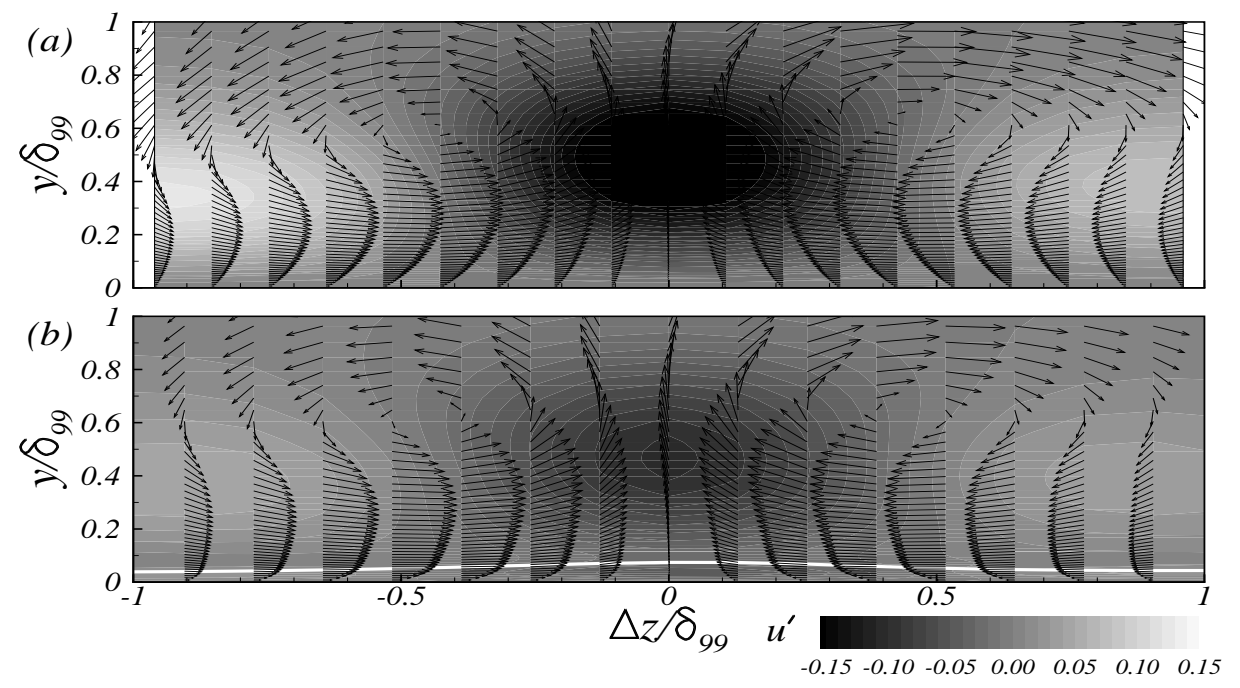

Figure 16. Conditional averages of flow fields for strong ejection $\left(-u^{\prime} v^{\prime}>2 u_{r m s}^{\prime} v_{r m s}^{\prime}, u^{\prime}<0\right.$ and $\left.v^{\prime}>0\right)$ at $x-x_{0}=200$ and $y / \delta_{99}=0.5$. (a) $\mu_{B T}=1.0$ and $(b) \mu_{B T}=0.2$. Here, white line shows the location of interface. 

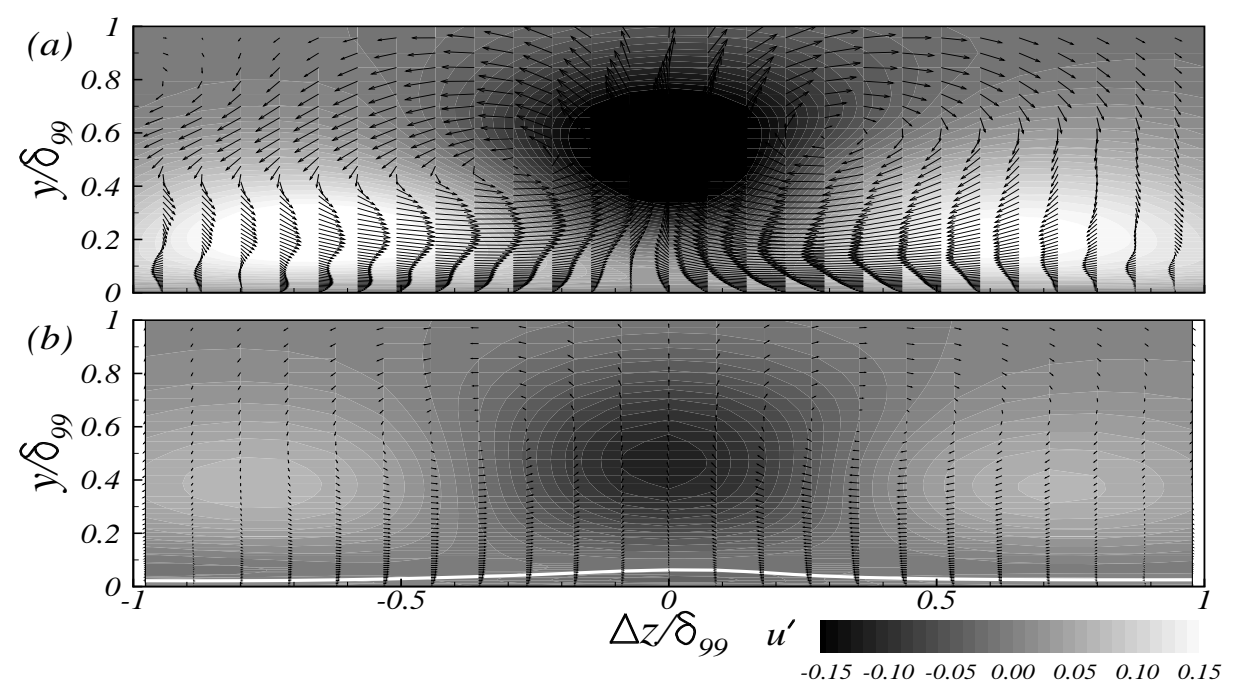

FiguRE 17. Conditional averages of flow fields for strong ejection $\left(-u^{\prime} v^{\prime}>2 u_{r m s}^{\prime} v_{r m s}^{\prime}, u^{\prime}<0\right.$ and $v^{\prime}>0$ ) at $x-x_{0}=400$ and $y / \delta_{99}=0.5$. ( $\left.a\right) \mu_{B T}=1.0$ and $(b) \mu_{B T}=0.2$. Here, white line shows the location of interface.

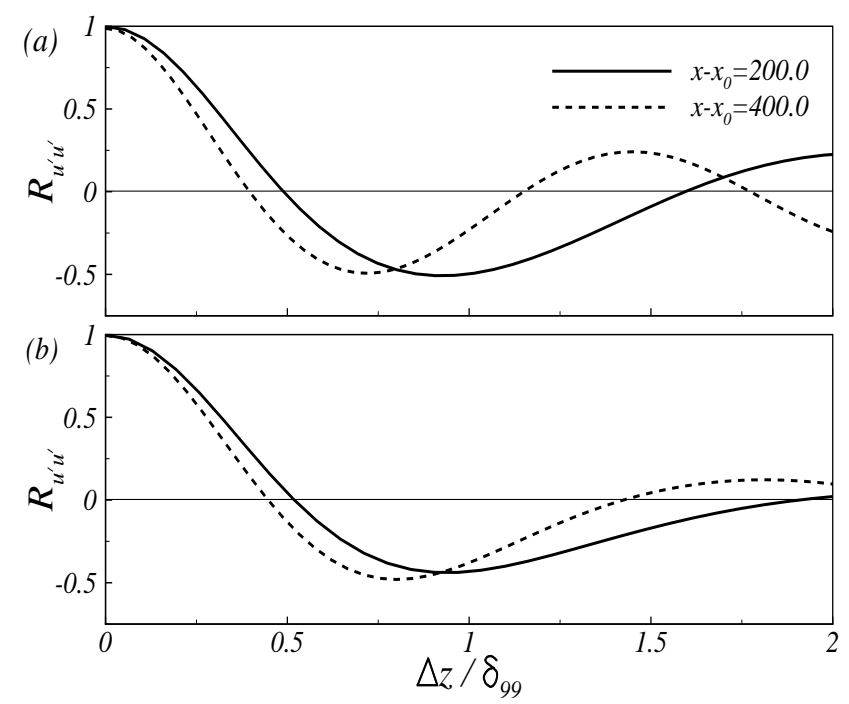

FIGURE 18. Spanwise correlations of streamwise velocity fluctuations at $y / \delta_{99}=0.5$ for $(a)$

$$
\mu_{B T}=1.0 \text { and }(b) \mu_{B T}=0.2 \text {. }
$$

In the conditionally-averaged fields, the spacing between secondary peaks becomes narrower downstream when scaled by the local boundary-layer thickness. This can be confirmed by the spanwise two-point correlation of the streamwise velocity fluctuations, $\overline{u^{\prime}(z+\Delta z) u^{\prime}(z)} / \overline{u^{\prime 2}}$, at $y / \delta_{99}=0.5$ as shown in figure 18 . The averaged streak spacing is defined as twice the distance to the minimum of the correlation. In local scaling, the spanwise spacing decreases slightly with downstream distance, which is consistent with the results by Brandt, Schlatter \& Henningson (2004).

The traditional statistical description of the streaky perturbation field provides $u_{r m s}^{\prime}$ and its maximum value (see figure 8). This approach masks the rich population of streak 

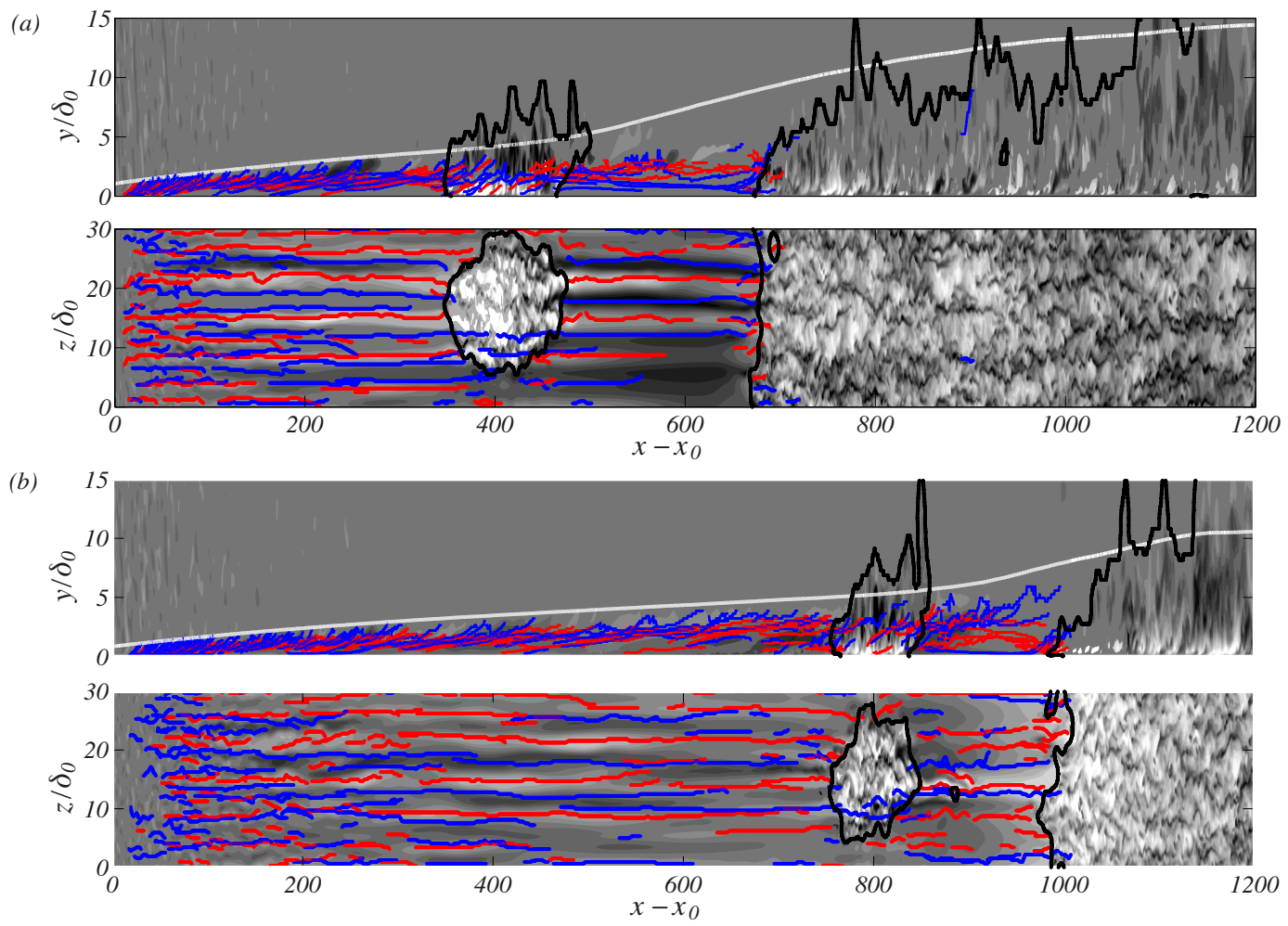

FIgURE 19. Snapshots of $u^{\prime}$ contours with positive (red) and negative (blue) streaks for $(a)$ $\mu_{B T}=1.0$ and $(b) \mu_{B T}=0.2$. Here, the black and white lines denote the laminar-turbulent edge detection and mean boundary layer thickness, respectively.

amplitudes which can deviate significantly from estimates based on $u_{r m s}^{\prime}$. It is therefore inadequate in predicting the impact of changes to the perturbation field on transition since breakdown is related to rare high-amplitude streaks. In order to more accurately characterize the pre-transitional boundary-layer, individual streaks must be identified in both space and time and appropriate statistical properties evaluated.

Here, we apply a recently introduced algorithm to isolate individual streaks in the DNS velocity fields (Nolan \& Zaki 2013), and report statistics of the streak population. The first step is to perform laminar-turbulence discrimination in order to isolate the pre-transitional flow. The discrimination algorithm is based on the techniques presented by Hedley \& Keffer (1974). The detector function adopted here is $D \equiv\left|v^{\prime}\right|+\left|w^{\prime}\right|$, which is a positive signal. The detector function is then low-pass filtered using a local standard-deviation filter, $\check{D}=G \star D$ where $G$ is the filtering Kernel. This step effectively homogenizes the laminar and turbulent regions to prepare the signal for effective laminarturbulence discrimination. It is important to recognize that the laminar and turbulent fields are two distinct dynamical systems that we wish to separate. Using a threshold $\check{D}_{t h}$, we define the indicator function, $\Gamma=1$ for $\check{D}>\check{D}_{t h}$, and $\Gamma=0$ otherwise. Otsu's method provides an effective approach to select the threshold (Otsu 1979). It was originally introduced for image processing, and is intended to separate the foreground and background of an image with bimodal distributions (e.g. range of light grays in the background and dark grays in the foreground). The method provides the optimal threshold which minimizes the total variance of the signal. The resulting logical indicator function, $\Gamma=\{0,1\}$, distinguishes the laminar and turbulent regions of the flow. Within the lami- 

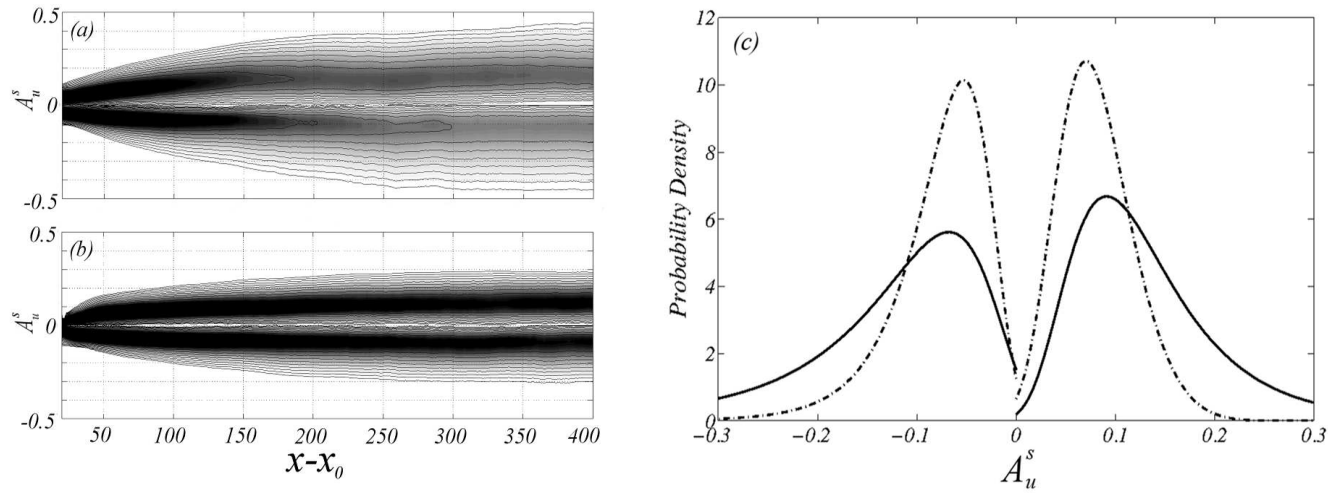

FiguRE 20. Streamwise variations of the distribution of $A_{u}^{s}$ for $(a) \mu_{B T}=1.0,(b) \mu_{B T}=0.2$ and (c) distributions of $A_{u}^{s}$ at $x-x_{0}=400$ for $\mu_{B T}=1.0$ (solid) and $\mu_{B T}=0.2$ (dash-dot). In figures $20(a)$ and $(b)$, contour lines are logarithmically distributed between decades $10^{-0.05}$ to 10 in steps of $10^{0.12}$.

nar region, individual streaks are identified in cross-stream planes by detecting the local peaks of the $u^{\prime}$ velocity. Maxima/minima located in the free stream or in the turbulent region of the boundary layer are discarded. Neighbouring maxima/minima in successive cross-stream planes are linked and identified as unique objects.

The laminar streaks extracted by the present detection algorithm are visualised in figure 19. The wall-normal and spanwise planes also show contours of streamwise velocity perturbations, $u^{\prime}$. The black line demarcates the interface between the laminar and turbulent regions of the flow. Red and blue lines indicate the spatial coordinates of the detected positive and negative streaks, respectively. The negative streaks are lifted towards the boundary layer edge, while positive streaks remain close to the wall. The turbulent spot enclosed by the black line appears further downstream when $\mu_{B T}=0.2$, which demonstrates the significant delay of bypass transition in the two-fluid boundary layer.

In order to assess the effect of introducing a wall-film on the amplitude of the laminar streaks, we track the streaks in time by cross-correlating their amplitudes in successive snapshots. For each streak, $s$, its coordinates $\mathbf{x}^{s}(t)$ describes its evolution in time, and its amplitude is defined as,

$$
\mathcal{A}_{u}^{s}\left(\mathbf{x}^{s}, t\right) \equiv u^{\prime}\left(\mathbf{x}=\mathbf{x}^{s}, t\right) .
$$

The maximum of $\mathcal{A}_{u}^{s}$ is extracted at every streamwise location,

$$
A_{u}^{s}(x)= \begin{cases}\max _{t, y, z}\left(\mathcal{A}_{u}^{s}\left(\mathbf{x}^{s}, t\right)\right), & \text { for } \mathcal{A}_{u}^{s}>0 \\ \min _{t, y, z}\left(\mathcal{A}_{u}^{s}\left(\mathbf{x}^{s}, t\right)\right), & \text { for } \mathcal{A}_{u}^{s}<0 .\end{cases}
$$

This expression identifies the maximum amplitude a particular streak, $s$, adopts during its entire time history as it passes an observation point, $x$. The associated wall-normal location is,

$$
y^{s}(x)=y\left(A_{u}^{s}(x)\right) .
$$

Figures $20 a$ and $20 b$ show the streamwise development of the distributions of $A_{u}^{s}$ in the single- and two-fluid boundary layers, respectively. In the case with $\mu_{B T}=1.0$, the amplitude of both positive and negative streaks grows quickly initially. Streak amplitudes attain a maximum value as high as $45 \%$ of the local free-stream velocity. The streamwise growth of the distribution is significantly inhibited in the case with $\mu_{B T}=0.2$ and the 


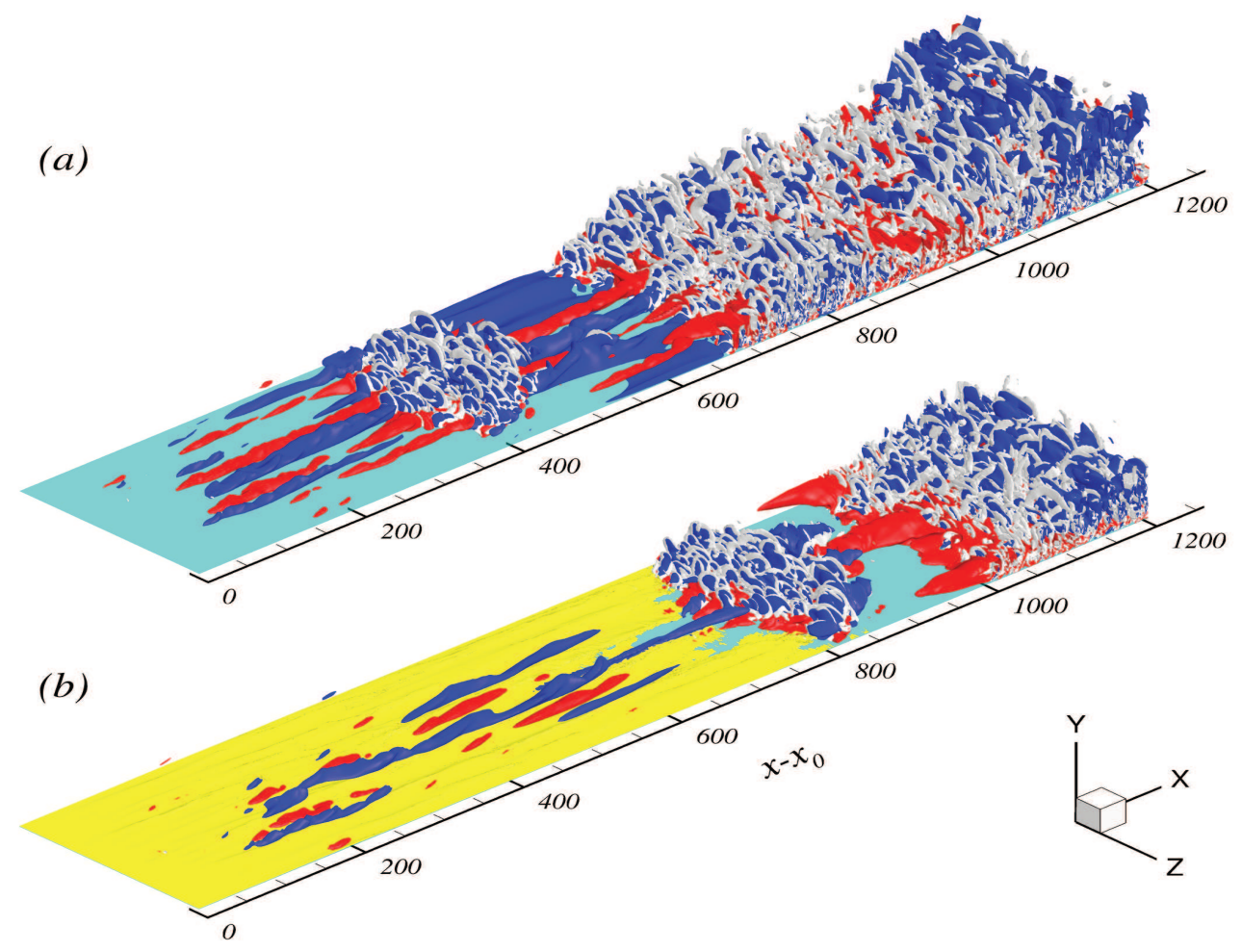

Figure 21. Snapshots of 3D vortical structure by $\mathrm{Q}$ criterion (white, $Q=0.001|Q|_{\max }$ ) with iso-surfaces of low-speed (blue, $u^{\prime}<-0.1 U_{\infty}$ ) and high-speed (red, $u^{\prime}>0.1 U_{\infty}$ ) streaks. Yellow contour for $\mu_{B T}=0.2$ denotes the location of interface. The wall-normal and spanwise extents are scaled by a factor of 8 . (a) $\mu_{B T}=1.0$ and $(b) \mu_{B T}=0.2$.

maximum amplitude is reduced by $30 \%$. The difference between the two cases at a fixed streamwise position upstream of transition is compared in figure $20 \mathrm{c}$. The majority of the streaks in the $\mu_{B T}=1.0$ case have an amplitude between $10 \%$ and $15 \%$. On the other hand, when $\mu_{B T}=0.2$, the amplitude of most streaks is confined to within $10 \%$. Highamplitude streaks $A_{u}>20 \%$, which are the common sites for breakdown to turbulence, are more frequent in the single-fluid boundary layer.

Figure 21 compares the instantaneous perturbation streaks and also the three-dimensional vortical structures within the single- and two-fluid boundary layers. The streaks are visualized using iso-surfaces of $u$-perturbations, and the vortices are educed using the $\mathrm{Q}$ criterion (Hunt, Wray \& Moin 1988). Here, Q is defined as $\mathrm{Q} \equiv-0.5\left(S_{i j}^{2}-\Omega_{i j}^{2}\right)$, where $S_{i j}$ and $\Omega_{i j}$ are the symmetric and anti-symmetric velocity gradient tensors, respectively. Abundant hairpin-like structures atop the low-speed streaks are clearly seen in the transitional region. The occurrence of turbulent spots takes place farther downstream in the two-fluid boundary layer, as explained by the weaker population of streaks in the pretransitional regime. The depletion of the wall film after $x-x_{0}=800$ in figure 21(b) is due to the strong velocity fluctuations in the transitional and the turbulent regions. In the former, the top-down turbulent spots impinge onto the wall and deplete the film. As a result, the computational cost of tracking the resulting droplets becomes prohibitive. The obliteration of the wall film is, however, downstream of transition and does not affect the underlying mechanism of transition delay in two-fluid boundary layers.

Transition length mainly depends on the rates of turbulent spot formation and spread- 

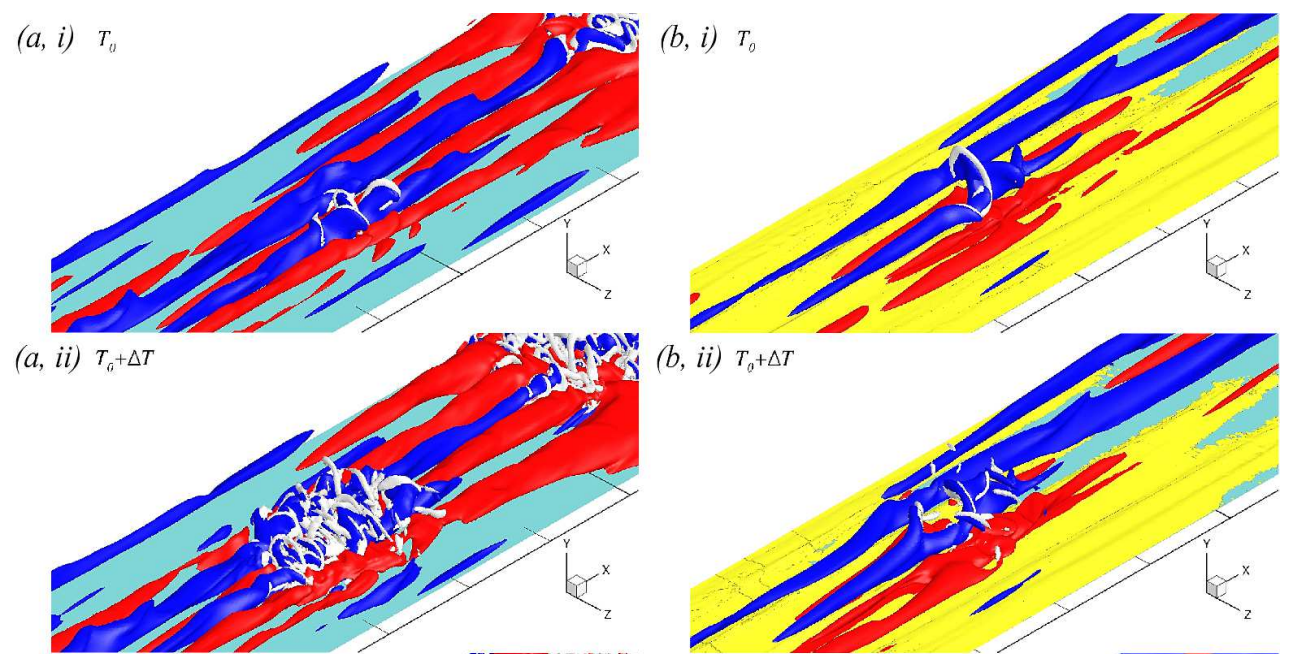

(b, ii) $T_{0}+\Delta T$
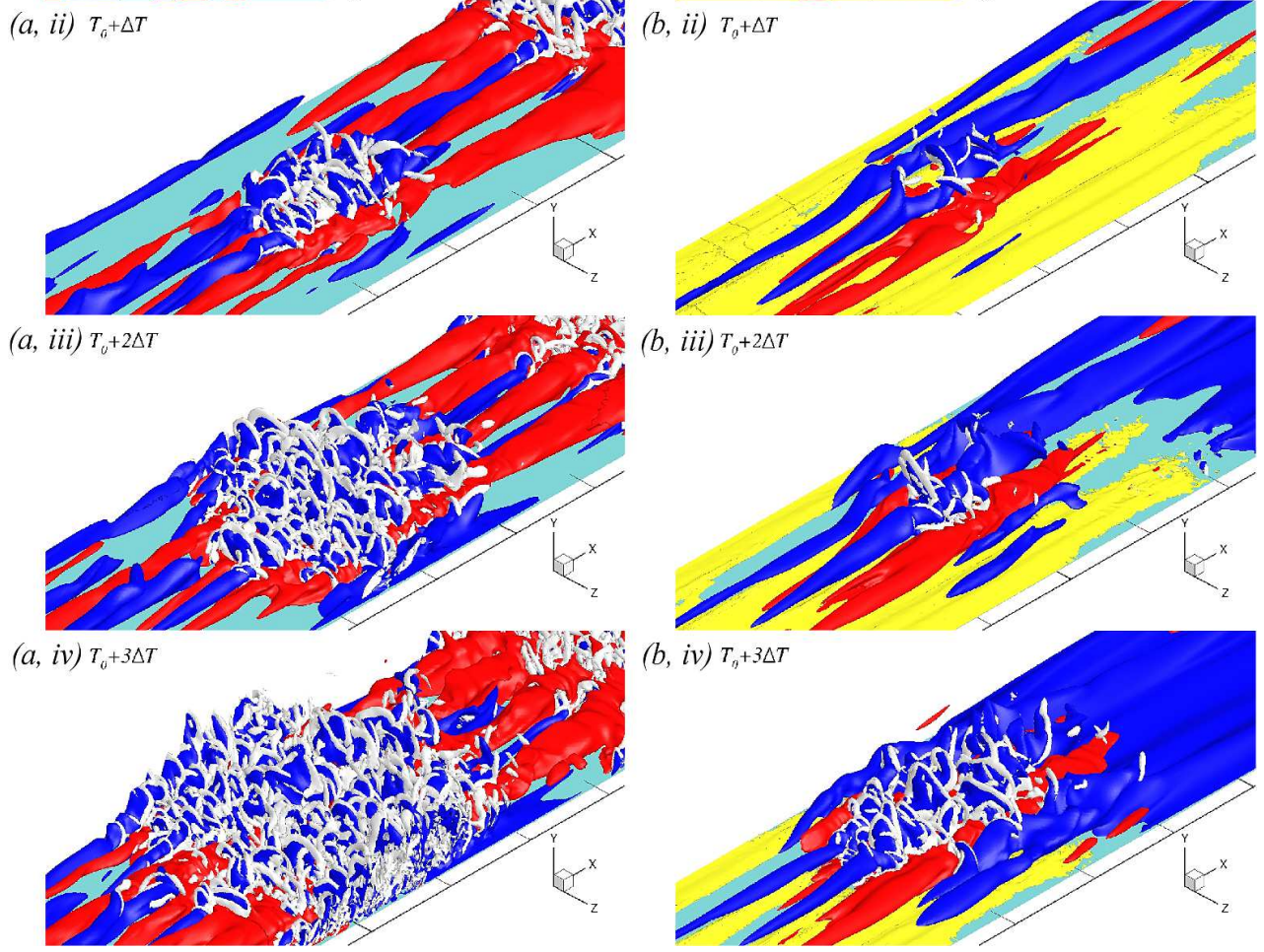

(b, iii) $T_{0}+2 \Delta T$

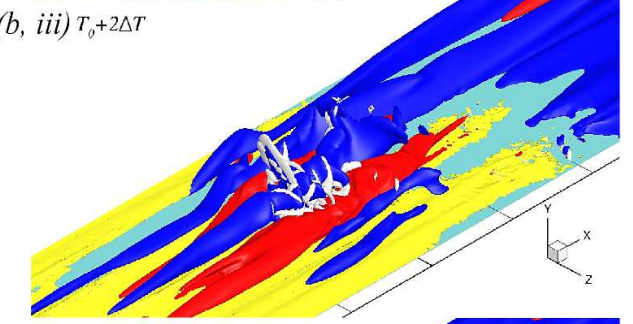

(b, iv) $T_{n}+3 \Delta T$

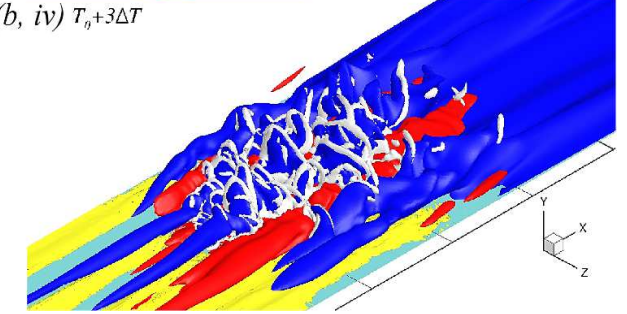

FIGURE 22. Time sequence of 3D vortical structure for $(a) \mu_{B T}=1.0$ and $(b) \mu_{B T}=0.2$. Here, $\Delta T=120 \delta_{0} / U_{\infty}$ and the viewing window is translated at $0.83 U_{\infty}$.

ing. A qualitative view of spot spreading in the single- and two-fluid boundary layers is captured by the time sequences in figures $22 a$ and $b$, respectively. It is clear in figure $22 a$ that the onset of the turbulent spot is closely linked to the strong negative streaks. As the turbulent patch convects downstream, it spreads longitudinally and laterally. Contrary to the single-fluid boundary layer, the spreading rate of the turbulent spots is much slower in the presence of the wall film as seen in figure $22 b$. This recalls the retarded development of the skin friction in figure 3, akin to a favourable-pressure-gradient boundary layer. The instantaneous flow fields presented so far provide convincing evidence of the impeded transition in the two-fluid boundary layer.

\section{Effect of higher turbulence intensity $(T u=3.5 \%)$}

In order to explore the effects of turbulence intensity on transition in two-fluid boundary layers, auxiliary simulations were performed $T u=3.5 \%$. As shown in table 1 , the streamwise domain size is shortened due to earlier transition at the higher turbulence intensity. 


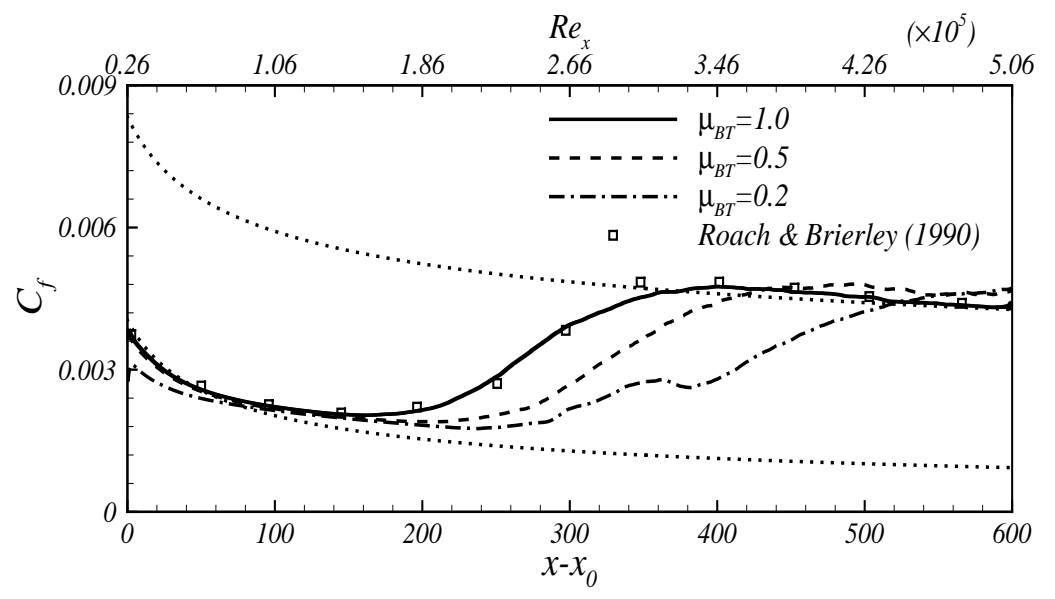

FiguRE 23. Downstream evolution of the skin friction coefficient for $T u=3.5 \%$.

The skin-friction coefficients for three viscosity ratios are plotted in figure 23 . It is possible to compare the single-fluid results to published data at this value of $T u \simeq 3.5 \%$ since it has been widely studied in the literature. In particular, the skin-friction coefficient from the experiments by Roach \& Brierley (1990) is included in figure 23 for comparison. Near the inlet, the two-fluid boundary layers have smaller $C_{f}$ values than the Blasius solution. Delay of transition is observed with decreasing viscosity of the wall film. The location of transition onset is $x-x_{0} \approx\{160,200,240\}$ for $\mu_{B T}=\{1.0,0.5,0.2\}$. Transition delay is inappreciable compared to the case of lower turbulence intensity $(T u=2.5 \%)$. The transition processes are complete by $x-x_{0} \approx 500$ for $\mu_{B T}=1.0$ and 0.5 . However, the lowest viscosity ratio case still shows juxtaposed regions of laminar and turbulent flows at the outlet.

The boundary-layer response to the high free-stream turbulence intensity is examined by evaluating the rms streamwise velocity fluctuations at several $x$-locations in figure 24 . The impaired amplification of the velocity fluctuations is clear with reduced viscosity ratio as in the $T u=2.5 \%$ case. Note that the inner peak of $\mu_{B T}=0.2$ in the laminar region protrudes due to the large jump in mean shear across the interface. The maximum value of $u_{r m s}^{\prime}$ for each viscosity ratio is shown in figure 25. It should be noted in figure 25(a) that the outer peak in the laminar region $\left(x-x_{0}<150\right)$ grows gently with decreasing film viscosity, which reflects the weakened streak amplification by the wall film. The outer peak reaches the maximum value at $x-x_{0}=290,350$ and 450 in case of $\mu_{B T}=1.0,0.5$ and 0.2 , respectively. In figure $25(\mathrm{~b})$, the inner peaks of two-fluid boundary layers only appear near the inlet region, $x-x_{0}<150$. Note also that the amplitude of the inner peak for $\mu_{B T}=0.2$ is larger than that of $\mu_{B T}=0.5$, consistent with earlier observations for $T u=2.5 \%$. In contrast to that case, however, the inner peak is overwhelmed by the outer perturbation field which amplifies quickly and causes breakdown to turbulence. The statistical measures from the higher level of free-stream turbulence demonstrate the delay of transition in the presence of the wall film. However, the effectiveness of the film in delaying transition is appreciably reduced. This reduction in the propensity of the film to delay transition is due to the stronger streak perturbation field in the outer flow, which is responsible for the onset of breakdown. A parallel can be drawn to the progressively weaker ability of favourable pressure gradient to delay bypass transition at higher $T u$. 


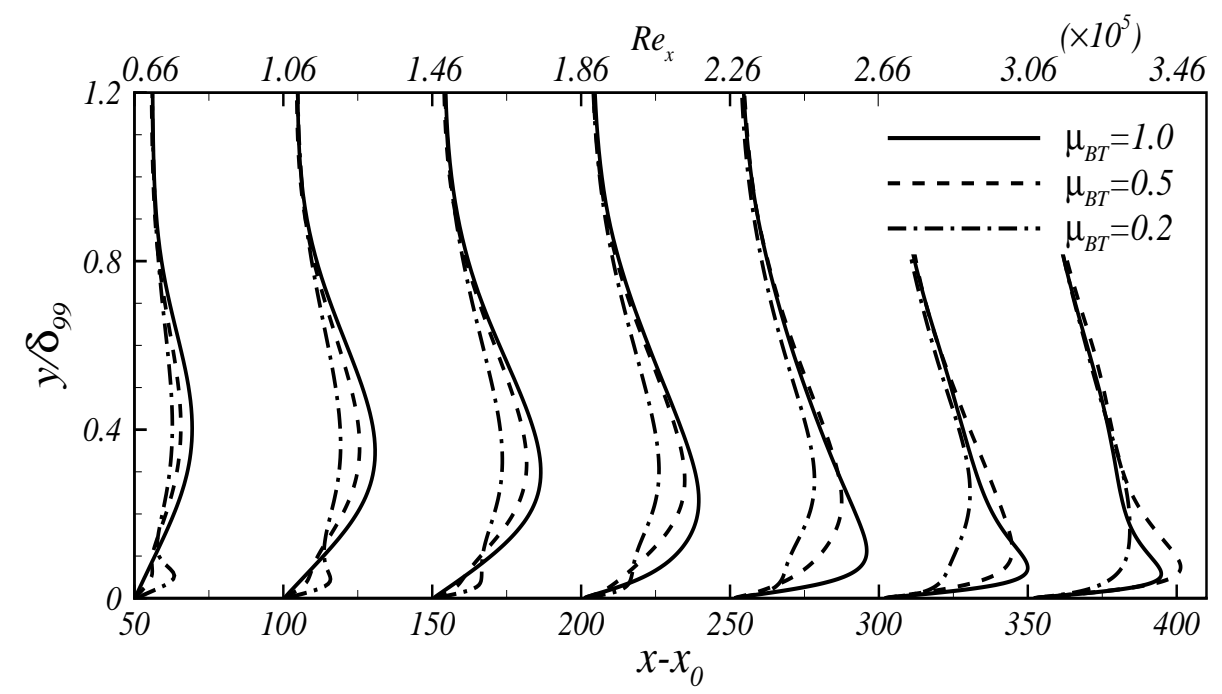

FiguRE 24. Profiles of the root-mean-square streamwise velocity fluctuations at various downstream locations. On the $x$-axis, the distance between major tick marks denotes $u_{r m s}^{\prime}=0.17 U_{\infty}$.

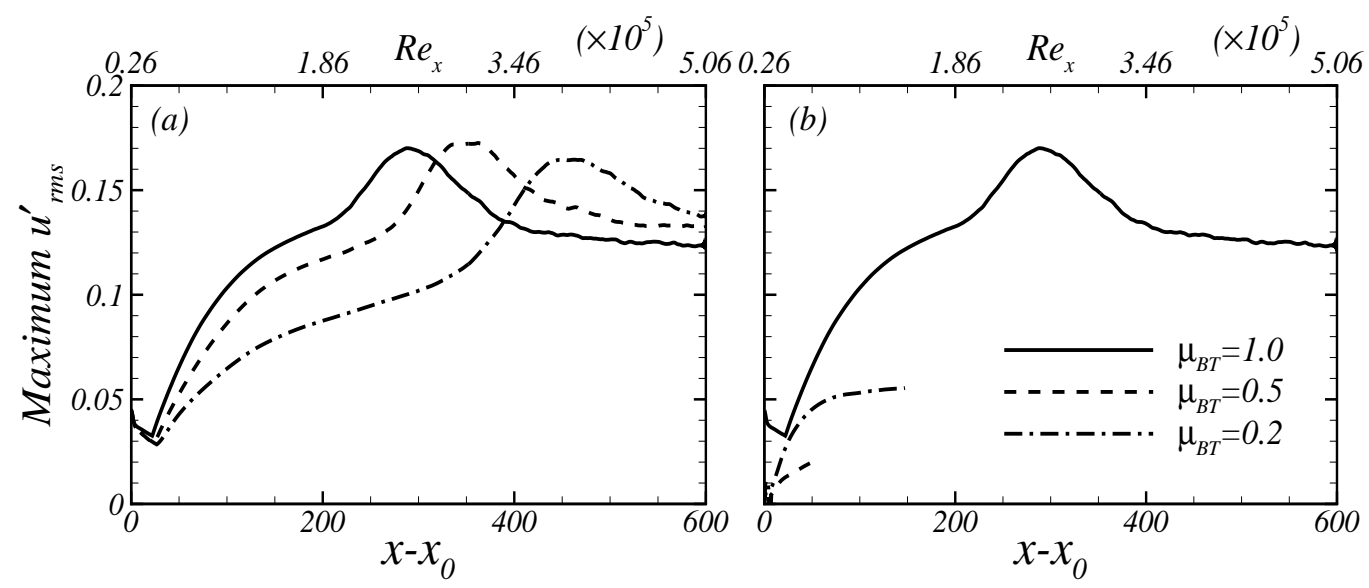

FiguRE 25. Maximum $u_{r m s}^{\prime}$ versus downstream distance for $(a)$ outer peak and $(b)$ inner peak.

\section{Summary}

Direct numerical simulations of two-fluid boundary layers beneath free-stream turbulence were performed in order to examine the influence of the wall film on bypass transition. Two moderate levels of free-stream turbulence intensities were considered, $T u=2.5$ and $3.5 \%$, with particular focus on the lower level. The thickness of the wall-film at the inlet to the computational domain was $d=10 \%$ of the single-fluid boundary-layer thickness at that location. Two wall-film-to-free-stream viscosity ratios were investigated, $\mu_{B T}=0.5$ and 0.2 , in addition to a reference single-fluid case. While this choice of viscosity ratios is motivated by previous linear studies, it should be noted that a lower viscosity film is not always guaranteed to delay transition: For example, the film can al- 
ter the receptivity of the boundary layer to free-stream disturbances (Zaki \& Saha 2009); the viscosity mismatch at the two-fluid interface can lead to new instability mechanisms (Yih 1967); and predictions based on linear theory become inaccurate when finite interface displacements are taken into consideration (Cheung \& Zaki 2010). Therefore, only direct numerical simulations can provide a complete account of the influence of a wall film on the full transition process.

In the present simulations, the wall film was effective at delaying laminar-to-turbulence transition. In particular, the increase in the skin-friction coefficient associated with the onset of turbulence was shifted downstream appreciably in the presence of the less viscous wall film. The cause for transition delay was examined using Reynolds-averaged statistics and analysis of instantaneous flow fields. The root-mean-squared streamwise velocity fluctuations were significantly weakened in the two-fluid boundary layer relative to the single-fluid configuration. The lower values of $u_{r m s}^{\prime}$ pointed to weaker Klebanoff streaks in the pre-transitional boundary layer. This effect was explained by appealing to the linear perturbation equations: The less viscous wall film reduces the mean shear in the outer fluid, which weakens the lift-up, or vorticity tilting, mechanism that generates the streaks. The equations also predict the presence of a second disturbance amplification mechanism at the two-fluid interface due to the viscosity mismatch, and which was observed in the numerical simulation.

The weakening of the streaks in the outer flow was also observed in the instantaneous flow fields. By identifying particular streaks in space and tracking them in time, probability distribution functions of the streak amplitudes were evaluated for each flow configuration. In the two-fluid case, the result showed a clear reduction in the amplitudes of the streaks within the population, and a lower frequency of occurrence of high-amplitude events which are the sites for secondary instability and the inception of turbulence spots. As a result, the secondary instabilities were less likely and the subsequent breakdown to turbulence occurred farther downstream.

The effectiveness of the wall film in delaying transition depends on the disturbance environment. When the turbulence intensity was increased to $T u=3.5 \%$, transition was still delayed in the two-fluid configuration. However, the extent of transition delay was less pronounced than at $T u=2.5 \%$. In addition, changes in the film properties can be anticipated to alter its performance. The present work considered a two-fluid boundary layer with a less viscous wall film - a configuration that can be emulated by heating of liquid flows at high Prandtl number (Lee et al. 2013). Future work can also examine the influence of density variation on the bypass transition process.

\section{REFERENCES}

Andersson, P., Brandt, L., Bottaro, A. \& Henningson, D. S. 2001 On the breakdown of boundary layer streaks. J. Fluid Mech. 428, 29-60.

Brandt, L., Schlatter, P. \& Henningson, D. S. 2004 Transition in boundary layers subject to free-stream turbulence. J. Fluid Mech. 517, 167-198.

Brodkey, R. S., Wallace, J. M. \& Eckelmann, H. 1974 Some properties of truncated turbulence signals in bounded shear flows. J. Fluid Mech. 63, 209-224.

Butler, K. M. \& Farrell, B. F. 1992 Three-dimensional optimal perturbations in viscous shear flow. Phys. Fluids A 4, 1637-1650.

Choi, H., Moin, P. \& Kim, J. 1994 Active turbulence control for drag reduction in wallbounded flows. J. Fluid Mech. 262, 75-110.

Charru, F. \& Hinch, E. J. 2000 Phase diagram of interfacial instabilities in a two-layer Couette flow and mechanism of the long-wave instability. J. Fluid Mech. 414, 195-223. 
Cheung, L. C. \& ZAKI, T. A. 2010 Linear and nonlinear instability waves in spatially developing two-phase mixing layers. Phys. Fluids 22, 052103.

Cheung, L. C. \& ZAKi, T. A. 2011 A nonlinear PSE method for two-fluid shear flows with complex interfacial topology J. Comput. Phys. 230, 6756-6777.

Desjardins, O., Moureau, V. \& Pitsch, H. 2008 An accurate conservative level set/ghost fluid method for simulating turbulent atomization. J. Comput. Phys. 227, 8395-8416.

Fukagata, K., Iwamoto, K. \& Kasagi, N. 2002 Contribution of Reynolds stress distribution to the skin friction in wall-bounded flows. Phys. Fluids 14, L73-L76.

Gueyffier, D., Li, J., Nadim, A., Scardovelli, R. \& Zaleski, S. 1999 Volume-of-fluid interface tracking with smoothed surface stress methods for three-dimensional flows. $J$. Comput. Phys. 152, 423-456.

HACK, M. J. P. \& ZAKI, T. A. 2014 Streak instabilities in boundary layers beneath free-stream turbulence. J. Fluid Mech. 741, 280-315.

Hedley, T. B. \& Keffer, J. F. 1974 Turbulent/non-turbulent decisions in an intermittent flow. J. Fluid Mech. 64, 625-644.

Hernon, D., Walsh, E. J. \& McEligot, D. M. 2007 Experimental investigation into the routes to bypass transition and the shear-sheltering phenomenon. J. Fluid Mech. 591, 461-479.

Hinch, E. J. 1984 A note on the mechanism of the instability at the interface between two shearing fluids. J. Fluid Mech. 144, 463-465.

Hooper, A. P. \& Boyd, W. G. C. 1987 Shear-flow instability due to a wall and a viscosity discontinuity at the interface. J. Fluid Mech. 179, 201-225.

Hunt, J. C. R. \& Durbin, P. A. 1999 Perturbed vortical layers and shear sheltering. Fluid Dyn. Res. 24 (6), 375-404.

Hunt, J. C. R., Wray, A. A. \& Moin, P. 1988 Eddies, streams, and convergence zones in turbulent flows. In Proceedings of Summer Program, 9-14, Centre for Turbulence Research, Stanford University.

Jacobs, R. G. \& Durbin, P. A. 1998 Shear sheltering and the continuous spectrum of the Orr-Sommerfeld equation. Phys. Fluids 10, 2006-2011.

Jacobs, R. G. \& Durbin, P. A. 2001 Simulations of bypass transition. J. Fluid Mech. 428, $185-212$.

KAng, H. S., Choi, H. \& Yoo, J. Y. 1998 On the modification of the near-wall coherent structure in a three-dimensional turbulent boundary layer on a free rotating disk. Phys. Fluids 10, 2315-2322.

Kim, J. \& Moin, P. 1985 Application of a fractional-step method to incompressible NavierStokes equations. J. Comput. Phys. 59, 308-323.

Kleiser, L. \& ZANG, T. A. 1991 Numerical simulation of transition in wall-bounded shear flows. Annu. Rev. Fluid Mech. 23, 495-537.

Kravchenko, G., Choi, H. \& Moin, P. 1993 On the relation of near-wall streamwise vortices to wall skin friction in turbulent boundary layers. Phys. Fluids A 5, 3307-3309.

Kurian, T. \& Fransson, J. H. M. 2009 Grid-generated turbulence revisited. Fluid Dyn. Res. 41, 021403.

Le, A. T., Coleman, G. N. \& Kim, J. 2000 Near-wall turbulence structures in threedimensional boundary layers. Int. J. Heat Fluid Flow 21, 480-488.

Lee, J., Jung, S. Y., Sung, H. J. \& ZAKi, T. A. 2013 Effect of wall heating on turbulent boundary layers with temperature-dependent viscosity. J. Fluid Mech. 726, 196-225.

Littell, H. S. \& Eaton, J. K. 1994 Turbulence characteristics of the boundary layer on a rotating disk. J. Fluid Mech. 266, 175-207.

Luchini, P. 2000 Reynolds-number-independent instability of the boundary layer over a flat surface: optimal perturbations. J. Fluid Mech. 404, 289-309.

Mandal, A. C., Venkatakrishnan, L. \& Dey, J. 2010 A study on boundary-layer transition induced by free-stream turbulence. J. Fluid Mech. 660, 114-146.

Malik, S. V. \& Hooper, A. P. 2007 Three-dimensional disturbances in channel flows. Phys. Fluids 19, 052102.

Matsubara, M. \& Alfredsson, P. H. 2001 Disturbance growth in boundary layers subjected to free-stream turbulence. J. Fluid Mech. 430, 149-168. 
Nelson, J. J., Alving, A. E. \& Joseph, D. D. 1995 Boundary layer flow of air over water on a flat plate. J. Fluid Mech. 284, 159-169.

Nagarajan, S., Lele, S. K. \& Ferziger, J. H. 2007 Leading-edge effects in bypass transition. J. Fluid Mech. 572, 471-504.

Nolan, K. P. \& WALsh, E. J. 2012 Particle image velocimetry measurements of a transitional boundary layer under free stream turbulence. J. Fluid Mech. 702, 215-238.

Nolan, K. P. \& ZAKI, T. A. 2013 Conditional sampling of transitional boundary layers in pressure gradients. J. Fluid Mech. 728, 306-339.

Nourgaliev, R. R. \& Theofanous T. G. 2007 High-fidelity interface tracking in compressible flows: Unlimited anchored adaptive level set. J. Comput. Phys. 224, 836-866.

Ó Náraigh, L., Spelt, P. D. M., Matar, O. K. \& Zaki, T. A. $2011 a$ Interfacial instability in turbulent flow over a liquid film in a channel. Int. J. Multiphase Flow 37, 812-830.

Ó Náraigh, L., Spelt, P. D. M. \& Zaki, T. A. $2011 b$ Turbulent flow over a liquid layer revisited: multi-equation turbulence modelling. J. Fluid Mech. 683, 357-394.

Osher, S. \& Sethian J. A. 1988 Fronts propagating with curvature-dependent speed: Algorithms based on Hamilton-Jacobi formulations. J. Comput. Phys. 79, 12-49.

Otsu, N. 1979 A threshold selection method from gray-level histograms. Systems, Man and Cybernetics, IEEE Transactions 9(1), 62-66.

Peng, D., Merriman, B., Osher, S., Zhao, H. \& Kang, M. 1999 A PDE-based fast local level set method. J. Comput. Phys. 155, 410-438.

Renardy, Y. 1987 The thin-layer effect and interfacial stability in a two-layer Couette flow with similar liquids. Phys. of Fluids 30, 1627-1637.

RoAch, P. E. \& Brierley, D. H. 1990 The influence of a turbulent freestream on zero pressure gradient transitional boundary layer development, part I: test cases T3A and T3b. ERCOFTAC Workshop: Numerical Simulation of Unsteady Flows and Transition to Turbulence, Lausanne, Switzerland, pp. 319-347. Cambridge University Press.

Rosenfeld, M., KwaK, D. \& Vinokur, M. 1991 A fractional step solution method for the unsteady incompressible Navier-Stokes equations in generalized coordinate systems. J. Comput. Phys. 94, 102-137.

SAHA, S., Jung, S. Y. \& ZAKI, T. A. 2010 Stabilization of wall shear beneath vortical disturbances using a thin film. In the 7th International Conference on Multiphase Flow, Tampa, USA.

Schlatter, P., Brandt, L., De Lange, H. C. \& Henningson, D. S. 2008 On streak breakdown in bypass transition. Phys. Fluids 20, 101505.

Sethian, J. A. \& Smereka, P. 2003 Level set methods for fluid interfaces. Annu. Rev. Fluid Mech. 35, 341-372.

SHu, C. -W. \& Osher, S. 1988 Efficient implementation of essentially non-oscillatory shockcapturing schemes. J. Comput. Phys. 77, 439-471.

VAN DER Vorst, H. A. 1992 Bi-CGSTAB: A fast and smoothly converging variant of Bi-CG for the solution of nonsymmetric linear systems. SIAM J. Sci. Stat. Comput. 13, 631-644.

VAN DER VORST, H. A. 2003 Iterative Krylov methods for large linear systems (first edition), Cambridge University Press.

Vaughan, N. J. \& ZAKI, T. A. 2011 Stability of zero-pressure-gradient boundary layer distorted by unsteady Klebanoff streaks. J. Fluid Mech. 681, 116-153.

Westin, K. J. A., Boiko, A. V., Klingmann, B. G. B., Kozlov, V. V. \& Alfredsson, P. H. 1994 Experiments in a boundary layer subjected to free stream turbulence. Part 1. Boundary layer structure and receptivity. J. Fluid Mech. 281, 193-218.

White, F. M. 2006 Viscous fluid flow (third edition), McGraw-Hill.

Willmarth, W. W. \& Lu, S. S. 1972 Structure of the Reynolds stress near the wall. J. Fluid Mech. 55, 65-92.

YANG, J. \& Stern, F. 2009 Sharp interface immersed-boundary/level-set method for wave-body interactions. J. Comput. Phys. 228, 6590-6616.

Yecko, P. \& Zaleski, S. 2005 Transient growth in two-phase mixing layers. J. Fluid Mech. 528, 43-52.

Yıн, C.-S. 1967 Instability due to viscosity stratification. J. Fluid Mech. 27, 337-352. 
ZAKi, T. A. \& Durbin, P. A. 2005 Mode interaction and the bypass route to transition. J. Fluid Mech. 531, 85-111.

Zaki, T. A. \& Durbin, P. A. 2006 Continuous mode transition and the effects of pressure gradient. J. Fluid Mech. 563, 357-388.

ZAKI, T. A. \& SAHA, S. 2009 On shear sheltering and the structure of vortical modes in singleand two-fluid boundary layers. J. Fluid Mech. 626, 111-147.

ZAKI, T. A. 2013 From Streaks to spots and on to turbulence: exploring the dynamics of boundary layer transition. Flow, Turb. ES Comb. 91, 451-473.

ZALEsAK, S. T. 1979 Fully multidimensional flux-corrected transport algorithms for fluids. J. Comput. Phys. 31, 335-362. 